\title{
Non-Archimedean Fuzzy M-Metric Space and Fixed Point Theorems Endowed with a Reflexive Digraph
}

\author{
Anuradha $^{1, *}$, Seema Mehra ${ }^{1}$, Said Broumi ${ }^{2}$ \\ ${ }^{1}$ Department of Mathematics, Maharshi Dayanand University (M.D.U), India \\ ${ }^{2}$ Faculty of Science Ben M'Sik, University Hassan II, Morocco
}

Received September 20, 2019; Revised November 5, 2019; Accepted November 16, 2019

\begin{abstract}
Copyright $@ 2019$ by authors, all rights reserved. Authors agree that this article remains permanently open access under
\end{abstract} the terms of the Creative Commons Attribution License 4.0 International License

\begin{abstract}
Motivated by the concepts of fuzzy metric and m-metric spaces, we introduced the notion of NonArchimedean fuzzy m-metric space which is an extension of partial fuzzy metric space. We present some examples in support of this new notion. Regarding this notion, its topological structure and some properties are specified simultaneously. At the end, some fixed point results are also provided.
\end{abstract}

Keywords M-Metric Space, Fuzzy Metric Space, Non-Archimedean Fuzzy M-Metric Space, Fixed Point, Graph

\section{Introduction}

Zadeh [11] was first who introduced the concept of fuzzy sets in 1965. In 1981, the idea of fuzzy contractive mappings was initially introduced by Heilpern [20] and proved some fixed point theorems for these fuzzy contractive mappings in metric linear spaces. In literature, a number of fixed point theorems for fuzzy contractive mappings have come into sight. Now a days, researchers have great interest in theory of fuzzy metric spaces. Study inquiry into discipline of fixed point theory on fuzzy metric spaces ([2, 5, 8, 15, 20-23] etc.) has been developed more frequently.

Asadi et al. [12] proposed the m-metric, an intended conception of a partial metric and provided some fixed point results in these m-metric spaces and after that Samer Assaf [18] reintroduced the m-metric presented in [18] and extended it to allow negative values. Topology given by Samer Assaf [18] for open balls in m- metric spaces was optimal but not optimal given by Asadi et al. [12].

The fixed point theory with graph is a very interesting approach in the field of research and has wide number of applications in other fields. In 2006, Espinola and Kirk [16] established some results on merging the fixed point theory and graph theory. In 2008, Jachymski [9] studied the Banach contraction principle in metric space with graph and gave an interesting approach in this direction. His work is considered as a reference in this domain. Motivated by the work of Jachymski, several authors established many results on various spaces endowed with graph.

\section{Materials and Methods}

Definition 2.1. [18] Let $X$ be any non-empty set and $m$ be real valued mapping on $\mathrm{X} \times \mathrm{X}$. Consider $m_{x, y}=\min \{\mathrm{m}(\mathrm{x}, \mathrm{x}), \mathrm{m}(\mathrm{y}, \mathrm{y})\}$. We say that $\mathrm{m}$ is an $\mathrm{m}$-metric on $\mathrm{X}$ if it satisfies the following axioms:

1. for all $\mathrm{x}, \mathrm{y} \in \mathrm{X}, m_{x, y} \leq \mathrm{m}(\mathrm{x}, \mathrm{y})$.

2. for all $x, y \in X, m(x, y)=m(y, x)$.

3. for all $x, y \in X, m(x, x)=m(x, y)=m(y, y)$ if and only if $\mathrm{x}=\mathrm{y}$

4. for all $\mathrm{x}, \mathrm{y}, \mathrm{z} \in \mathrm{X}$, $\mathrm{m}(\mathrm{x}, \mathrm{y})-m_{x, y} \leq\left\{\mathrm{m}(\mathrm{x}, \mathrm{z})-m_{x, z}\right\}+\{\mathrm{m}(\mathrm{z}, \mathrm{y})-$ $\left.m_{z, y}\right\}$ Then the pair $(\mathrm{X}, \mathrm{m})$ is known as $\mathrm{m}$ - metric space.

Example 2.2. [18] Let $\mathrm{m}: \mathrm{R} \times \mathrm{R} \rightarrow \mathrm{R}$ be a mapping defined as $\mathrm{m}(\tau, \eta)=\tau+\eta$ for all $\tau, \eta \in \mathrm{R}$ then $\mathrm{m}$ is a $\mathrm{m}$ - metric on $\mathrm{R}$.

Definition 2.3.[21] A binary operation * : [0,1] $\times[0,1]$ $\rightarrow[0,1]$ is said to be continuous t-norm if it satisfies the following conditions:

1. * is associative and commutative;

2. $*$ is continuous;

3. $\eta * 1=1=1 * \eta \forall \eta \in[0,1]$;

4. $\tau * \eta \leq \kappa * \rho$ if $\tau \leq \kappa$ and $\eta \leq \rho$ for all $\tau, \kappa, \eta$, $\rho \in[0,1]$.

Definition 2.4. [21] A fuzzy metric ( in the sense George and Veeramani) on a nonempty set $\mathrm{X}$ is a function $\mathrm{M}: \mathrm{X} \times$ $\mathrm{X} \times(0, \infty) \rightarrow[0,1]$ such that for all $\tau, \eta, \kappa \in \mathrm{X}$ and $\mathrm{t}, \mathrm{s}>$ 0 ; 
1. $\mathrm{M}(\tau, \eta, \mathrm{t})>0$

2. $\tau=\eta \Leftrightarrow \mathrm{M}(\tau, \eta, \mathrm{t})=1$;

3. $\mathrm{M}(\tau, \eta, \mathrm{t})=\mathrm{M}(\eta, \tau, \mathrm{t})$;

4. $\quad \mathrm{M}(\tau, \eta, \mathrm{t}+\mathrm{s}) \geq \mathrm{M}(\tau, \kappa, \mathrm{t}) * \mathrm{M}(\kappa, \eta, \mathrm{s})$

5. $\mathrm{M}(\tau, \kappa, \cdot):(0, \infty) \rightarrow(0,1]$ is continuous mapping.

Then 3-tuple $(\mathrm{X}, \mathrm{M}, *)$ is called a fuzzy metric space where $*$ is a continuous t-norm. If the condition (4) is replaced by $\mathrm{M}(\tau, \eta, \max \{\mathrm{t}, \mathrm{s}\}) \geq \mathrm{M}(\tau, \kappa, \mathrm{t}) * \mathrm{M}(\kappa, \eta, \mathrm{s})$ then the space $(\mathrm{X}, \mathrm{M}, *)$ is called a non-Archimedian fuzzy metric space. Any non-Archimedian fuzzy metric space is a fuzzy metric space.

Definition 2.5.[21] A partial fuzzy metric is a ordered tuple $(\mathrm{X}, \mathrm{P}, *)$ such that $\mathrm{X}$ is a non-empty set, $*$ is a continuous t- norm and $\mathrm{P}$ is a fuzzy set on $\mathrm{X} \times \mathrm{X} \times(0, \infty)$ satisfying the following conditions, for all $\tau, \eta, \kappa \in \mathrm{X}$ and $\mathrm{t}, \mathrm{s}>0$

$(\mathrm{PFM}-1) \tau=\eta \Leftrightarrow \mathrm{P}(\tau, \tau, \mathrm{t})=\mathrm{P}(\tau, \eta, \mathrm{t})=\mathrm{P}(\eta, \eta, \mathrm{t})$

$(\operatorname{PFM}-2) \mathrm{P}(\eta, \eta, \mathrm{t}) \geq \mathrm{P}(\tau, \eta, \mathrm{t})$

$(\mathrm{PFM}-3) \mathrm{P}(\tau, \eta, \mathrm{t})=\mathrm{P}(\eta, \tau, \mathrm{t})$;

$(\mathrm{PFM}-4) \mathrm{P}(\tau, \eta, \max \{\mathrm{t}, \mathrm{s}\}) * \mathrm{P}(\kappa, \kappa, \max \{\mathrm{t}, \mathrm{s}\})$ $\geq \mathrm{P}(\tau, \kappa, \mathrm{t}) * \mathrm{P}(\kappa, \eta, \mathrm{s})$

$(\mathrm{PFM}-5) \mathrm{P}(\tau, \kappa, \cdot):(0, \infty) \rightarrow(0,1]$ is continuous.

It can be easily shown that if $\mathrm{P}(\tau, \eta, \mathrm{t})=1$, then from (PM-1) and (PM-2), we get $\tau=\eta$. But if $\tau=\eta, \mathrm{P}(\tau, \eta, \mathrm{t})$ may not be 1 . The 3 -tuple $\left(\mathrm{R}^{+}, \mathrm{P}, *\right)$, where $\mathrm{P}(\tau, \eta, \mathrm{t})=\frac{t}{t+\max \{\tau, \eta\}}$ is the simplest example of a partial fuzzy metric space (PFMS).

Example 2.6.[21] Let (X, p) be a partial metric space and $\mathrm{P}: \mathrm{X} \times \mathrm{X} \times(0, \infty) \rightarrow[0,1]$ be a mapping defined as

$$
\mathrm{P}(\tau, \eta, \mathrm{t})=\frac{t}{t+\mathrm{p}(\tau, \eta)} \text { or } \mathrm{P}(\tau, \eta, \mathrm{t})=e^{-\frac{p(\tau, \eta)}{t}} \text {. }
$$

If $\tau * \eta=\tau \eta$ for all $\tau, \eta \in[0,1]$, then clearly $\mathrm{P}$ is a partial fuzzy metric, but it may not be a fuzzy metric. For more details concerning partial fuzzy metric space, see [21].

Now we recall some basic concepts related to Graph theory.

Let $(\mathrm{X}, \mathrm{d})$ be a complete metric space. Throughout this paper, we suppose that $G=(\mathrm{V}, \mathrm{E})$ is a digraph such that $\mathrm{V}$ $(G)=X$ is the vertex set and $E(G)$ is the set of its edges contains no parallel edges with $(v, v) \in E(G)$. By ${ }^{-1}$, we mean that it is the inverse of the graph which is obtained from $G$ by reversing the direction of edges. By disregarding the direction of edges of $\mathrm{G}$, we acquire $\tilde{G}$, the undirected graph from $G$ so, $\mathrm{E}(\widetilde{G})=\mathrm{E}(\mathrm{G}) \cup \mathrm{E}\left(\mathrm{G}^{-1}\right)$. Actually, it will be more convenient for us to treat $\tilde{G}$ as a digraph for which the set of its edges is symmetric.

In this paper, motivated by the work of Sedghi et al. [21] and approach of fixed point theory with graph, we establish the notion of non-Archimedean fuzzy m-metric space. We also obtain their properties, its topological structure and some fixed point results in the context of non-Archimedean fuzzy m-metric space. Some of our results are generalization of Sedghi et al. [21]. These results generalize and subsume many previously obtained fixed point results.

In this paper, we organize our work in the following manner:

In section 3, we establish notion of Non-Archimedean fuzzy m-metric space, their properties and its topological structure then in section 4, present some fixed point theorems in context of non- Archimedean fuzzy m-metric space.

\section{Non-Archimedean Fuzzy M-Metric Space (NAFmMS) and Its Topology}

Here, we will define the notion of non-Archimedean fuzzy m-metric space and its topological properties.

Definition 3.1. Let $X$ be any non-empty arbitrary set,* be a continuous t-norm and mapping $\mathrm{F}_{\mathrm{m}}: \mathrm{X} \times \mathrm{X} \times(0, \infty) \rightarrow[0,1]$ satisfying, $\forall \tau, \eta, \kappa \in \mathrm{X}$ and $\mathrm{t}, \mathrm{s}>0$

(FMM 1) $\mathrm{F}_{\mathrm{m}}(\tau, \eta, \mathrm{t})=\mathrm{F}_{\mathrm{m}}(\eta, \eta, \mathrm{t})=\mathrm{F}_{\mathrm{m}}(\tau, \tau, \mathrm{t})$ if and only if $\tau=\eta$;

(FMM 2) $\max \left\{\mathrm{F}_{\mathrm{m}}(\tau, \tau, \mathrm{t}), \mathrm{F}_{\mathrm{m}}(\eta, \eta, \mathrm{t})\right\} \geq \mathrm{F}_{\mathrm{m}}(\tau, \eta, \mathrm{t})$;

(FMM 3) $\mathrm{F}_{\mathrm{m}}(\tau, \eta, \mathrm{t})=\mathrm{F}_{\mathrm{m}}(\eta, \tau, \mathrm{t})$;

(FMM 4) $\mathrm{F}_{\mathrm{m}}(\tau, \eta, \max \{\mathrm{t}, \mathrm{s}\}) * M_{\tau, \kappa, t, s} * M_{\eta, \kappa, t, s}$ $\geq \mathrm{F}_{\mathrm{m}}(\tau, \kappa, \mathrm{t}) * \mathrm{~F}_{\mathrm{m}}(\kappa, \eta, \mathrm{s}) * M_{\tau, \eta, t, s}$

(FMM 5) $\mathrm{F}_{\mathrm{m}}(\tau, \eta, \cdot):(0, \infty) \rightarrow[0,1]$ is continuous; where $M_{x, y, t, s}$ represents that

$M_{x, y, t, s}=\max \left\{\mathrm{F}_{\mathrm{m}}(\mathrm{x}, \mathrm{x}, \max \{\mathrm{t}, \mathrm{s}\}), \mathrm{F}_{\mathrm{m}}(\mathrm{y}, y, \max \{\mathrm{t}, \mathrm{s}\})\right\}$

$$
\forall x, y \in \mathrm{X}
$$

then $F_{m}$ is called Fuzzy m-metric on $X$ and the triplet $\left(\mathrm{X}, \mathrm{F}_{\mathrm{m}}, *\right)$ known as non-Archimedean fuzzy m-metric space (NAFmMS).

Since $F_{m}(x, x, \max \{t, s\}), F_{m}(y, y, \max \{t, s\}) \in[0,1]$, therefore $M_{x, y, t, s}$ exists for all $x, y \in \mathrm{X}$ and $\mathrm{t}, \mathrm{s}>0$. It is clear from (FMM 1), if $\tau=\eta$ then $\mathrm{F}_{\mathrm{m}}(\tau, \eta$, t) may not be 1 , also if $\mathrm{F}_{\mathrm{m}}(\tau, \eta, \mathrm{t})=1$ doesn't implies $\tau=\eta$ but $\mathrm{F}_{\mathrm{m}}(\tau, \eta, \mathrm{t})=1$ implies $\mathrm{F}_{\mathrm{m}}(\tau, \eta, \mathrm{t})=M_{\tau, \eta, t}$.

From (FMM 4), obviously we can observe that for all $\tau$, $\eta, \kappa \in \mathrm{X}$ and $\mathrm{t}>0$,

(FMM 6) $\mathrm{F}_{\mathrm{m}}(\tau, \eta, \mathrm{t}) * M_{\tau, \kappa, t} * M_{\kappa, \eta, t}$

$$
\geq \mathrm{F}_{\mathrm{m}}(\tau, \kappa, \mathrm{t}) * \mathrm{~F}_{\mathrm{m}}(\kappa, \eta, \mathrm{t}) * M_{\tau, \eta, t}
$$

where $M_{\tau, \eta, t}=\max \left\{\mathrm{F}_{\mathrm{m}}(\tau, \tau, \mathrm{t}), \mathrm{F}_{\mathrm{m}}(\eta, \eta, \mathrm{t})\right\} \forall \tau, \eta \in \mathrm{X}$. Also here, $M_{\tau, \eta, t}$ exist $\forall \tau, \quad \eta \in \mathrm{X}$ and $\mathrm{t}>0$ since $\mathrm{F}_{\mathrm{m}}(\tau, \tau, \mathrm{t}), \mathrm{F}_{\mathrm{m}}(\eta, \eta, \mathrm{t}) \in[0,1]$.

Some basic examples of non-Archimedean fuzzy $\mathrm{m}$-metric are given below:

Example 3.1. Let $X=\{\tau, \eta\}$ be a two point set where $\tau, \eta \in \mathrm{R}$ (set of reals) and $\mathrm{m}(\tau, \eta)$ be a m-metric on $\mathrm{X}$. For all $\tau, \eta \in \mathrm{X}$, the m-metric defined as $\mathrm{m}(\tau, \tau)=\mathrm{m}(\tau, \eta)=$ $\mathrm{m}(\eta, \tau)=1, \mathrm{~m}(\eta, \eta)=2($ not a partial metric $)$. Then mapping defined by $\mathrm{F}_{\mathrm{m}}(\tau, \eta, \mathrm{t})=1-e^{\frac{-m(\tau, \eta)}{t}}$ is a 
non-Archimedean fuzzy m-metric on $\mathrm{X}$ with $\tau * \eta=$ $\min \{\tau, \eta\}$.

Clearly, $\mathrm{F}_{\mathrm{m}}(\tau, \eta, \mathrm{t}) \in[0,1]$ and continuous in $\mathrm{t}$ and satisfying all the properties of fuzzy m-metric as

1. $\mathrm{F}_{\mathrm{m}}(\tau, \tau, \mathrm{t})=\mathrm{F}_{\mathrm{m}}(\eta, \eta, \mathrm{t})=\mathrm{F}_{\mathrm{m}}(\tau, \eta, \mathrm{t})$ if and only if $\tau=$ $\eta$, but $\mathrm{F}_{\mathrm{m}}(\tau, \tau, \mathrm{t})=\mathrm{F}_{\mathrm{m}}(\eta, \eta, \mathrm{t})=\mathrm{F}_{\mathrm{m}}(\tau, \eta, \mathrm{t})$, it is not possible.

2. $\max \left\{\mathrm{F}_{\mathrm{m}}(\tau, \tau, \mathrm{t}), \mathrm{F}_{\mathrm{m}}(\eta, \eta, \mathrm{t})\right\} \geq \mathrm{F}_{\mathrm{m}}(\tau, \eta, \mathrm{t})$, that is, $\max \left\{1-e^{\frac{-1}{t}}, 1-e^{\frac{-2}{t}}\right\} \geq 1-e^{\frac{-1}{t}}$ implies $1-e^{\frac{-2}{t}} \geq 1-e^{\frac{-1}{t}}$ which is true.

3. $\mathrm{F}_{\mathrm{m}}(\tau, \eta, \mathrm{t})=\mathrm{F}_{\mathrm{m}}(\eta, \tau, \mathrm{t}) \forall \tau, \eta \in \mathrm{X}$.

4. Let $\mathrm{t}>\mathrm{s}$, for $\kappa=\tau$

$\mathrm{F}_{\mathrm{m}}(\tau, \eta, \mathrm{t}) * M_{\tau, \kappa, t} * M_{\kappa, \eta, t} \geq \mathrm{F}_{\mathrm{m}}(\tau, \kappa, \mathrm{t}) * \mathrm{~F}_{\mathrm{m}}(\kappa, \eta, \mathrm{t}) *$ $M_{\tau, \eta, t}$

that is, $\left(1-e^{\frac{-1}{t}}\right) * \max \left\{1-e^{\frac{-1}{t}}, 1-e^{\frac{-1}{t}}\right\} * \max \left\{1-e^{\frac{-1}{t}}\right.$, $\left.1-e^{\frac{-2}{t}}\right\} \geq\left(1-e^{\frac{-1}{t}}\right) *\left(1-e^{\frac{-1}{t}}\right) * \max \left\{1-e^{\frac{-1}{t}}, 1-e^{\frac{-2}{t}}\right\}$ implies that

$\min \left\{\left(1-e^{\frac{-1}{t}}\right),\left(1-e^{\frac{-1}{t}}\right),\left(1-e^{\frac{-2}{t}}\right)\right\} \geq$ $\min \left\{\left(1-e^{\frac{-1}{t}}\right),\left(1-e^{\frac{-1}{t}}\right),\left(1-e^{\frac{-2}{t}}\right)\right\}$

which is also true.

Similarly if we take $\kappa=\eta$ then it is also hold.

Example 3.2. Suppose that $\mathrm{m}(\tau, \eta)$ be any m-metric on $\mathrm{X}$ and $\mathrm{F}_{\mathrm{m}}: \mathrm{X} \times \mathrm{X} \times(0, \infty) \rightarrow[0,1]$ is a mapping defined as

$$
\mathrm{F}_{\mathrm{m}}(\tau, \eta, \mathrm{t})=e^{\frac{-m(\tau, \eta)}{t}} .
$$

Then $\mathrm{F}_{\mathrm{m}}$ is a non-Archimedean fuzzy m-metric with $\tau * \eta=\eta, \forall \tau, \eta \in[0,1]$ but may not be a partial fuzzy metric.

Since, it is easy to prove the conditions (FMM 1), (FMM 3) and (FMM 5) of definition 3.1. by using concept of m-metric. Next, we have to prove the conditions (FMM 2) and (FMM 4) of definition 3.1.

(FMM 2) In m-metric space (X, m), we have

$$
\min \{\mathrm{m}(\tau, \tau), \mathrm{m}(\eta, \eta)\} \leq \mathrm{m}(\tau, \eta)
$$

Here two cases arise:

Case I: $\min \{\mathrm{m}(\tau, \tau), \mathrm{m}(\eta, \eta)\}=\mathrm{m}(\tau, \tau) \leq \mathrm{m}(\tau, \eta)$

$$
\Rightarrow \mathrm{F}_{\mathrm{m}}(\tau, \tau, \mathrm{t})=e^{\frac{-m(\tau, \tau)}{t}} \geq e^{\frac{-m(\tau, \eta)}{t}}=\mathrm{F}_{\mathrm{m}}(\tau, \eta, \mathrm{t}) .
$$

If $\mathrm{m}(\eta, \eta) \geq \mathrm{m}(\tau, \eta)$ then

$$
\mathrm{F}_{\mathrm{m}}(\eta, \eta, \mathrm{t})=e^{\frac{-m(\eta, \eta)}{t}} \leq e^{\frac{-m(\tau, \eta)}{t}}=\mathrm{F}_{\mathrm{m}}(\tau, \eta, \mathrm{t})
$$

implies that $\max \left\{\mathrm{F}_{\mathrm{m}}(\tau, \tau, \mathrm{t}), \mathrm{F}_{\mathrm{m}}(\eta, \eta, \mathrm{t})\right\}=\mathrm{F}_{\mathrm{m}}(\tau, \tau, \mathrm{t}) \geq$ $\mathrm{F}_{\mathrm{m}}(\tau, \eta, \mathrm{t})$.

Case II: $\min \{\mathrm{m}(\tau, \tau), \mathrm{m}(\eta, \eta)\}=\mathrm{m}(\eta, \eta) \leq \mathrm{m}(\tau, \eta)$

Similarly, it can be proved as in case I.

(FMM 4) Without loss of generality (W.L.G.), we take $t>$ s.

Again, in m-metric space we have

$$
\mathrm{m}(\tau, \eta)-m_{\tau, \eta} \leq \mathrm{m}(\tau, \kappa)-m_{\tau, \kappa}+\mathrm{m}(\kappa, \eta)-m_{\eta, \kappa}
$$

where $m_{\tau, \eta}=\min \{\mathrm{m}(\tau, \tau), \mathrm{m}(\eta, \eta)\}$

that is, $\mathrm{m}(\tau, \eta)+m_{\tau, \kappa}+m_{\eta, \kappa} \leq \mathrm{m}(\tau, \kappa)+\mathrm{m}(\kappa, \eta)+m_{\tau, \eta}$.
It is enough to take the following cases:

$$
\begin{aligned}
& \text { 1. } \mathrm{m}(\tau, \tau)=\mathrm{m}(\eta, \eta)=\mathrm{m}(\kappa, \kappa) \\
& \text { 2. } \mathrm{m}(\tau, \tau)<\mathrm{m}(\eta, \eta)<\mathrm{m}(\kappa, \kappa) \\
& \text { 3. } \mathrm{m}(\tau, \tau)<\mathrm{m}(\eta, \eta)=\mathrm{m}(\kappa, \kappa) \\
& \text { 4. } \mathrm{m}(\tau, \tau)>\mathrm{m}(\eta, \eta)=\mathrm{m}(\kappa, \kappa) \\
& \text { 5. } \mathrm{m}(\tau, \tau)=\mathrm{m}(\eta, \eta)<\mathrm{m}(\kappa, \kappa) \\
& \text { 6. } \mathrm{m}(\tau, \tau)=\mathrm{m}(\eta, \eta)>\mathrm{m}(\kappa, \kappa)
\end{aligned}
$$

Consider the case 2: $\mathrm{m}(\tau, \tau)<\mathrm{m}(\eta, \eta)<\mathrm{m}(\kappa, \kappa)$ then $\mathrm{m}(\tau, \eta)+m_{\tau, \kappa}+m_{\eta, \kappa} \leq \mathrm{m}(\tau, \kappa)+\mathrm{m}(\kappa, \eta)+m_{\tau, \eta}$ becomes

$$
\begin{gathered}
\mathrm{m}(\tau, \eta)+\mathrm{m}(\tau, \tau)+\mathrm{m}(\eta, \eta) \leq \mathrm{m}(\tau, \kappa)+\mathrm{m}(\kappa, \eta)+\mathrm{m}(\tau, \tau) \\
\Rightarrow e^{\frac{-(\mathrm{m}(\tau, \eta)+\mathrm{m}(\tau, \tau)+\mathrm{m}(\eta, \eta))}{t}} \geq e^{\frac{-(\mathrm{m}(\tau, \kappa)+\mathrm{m}(\kappa, \eta)+\mathrm{m}(\tau, \tau))}{t}} \\
\Rightarrow e^{\frac{-\mathrm{m}(\tau, \eta)}{t}} \cdot e^{\frac{-\mathrm{m}(\tau, \tau)}{t}} \cdot e^{\frac{-\mathrm{m}(\eta, \eta)}{t}} \geq e^{\frac{-\mathrm{m}(\tau, \kappa)}{t}} \\
\cdot e^{\frac{-\mathrm{m}(\kappa, \eta)}{t}} \cdot e^{\frac{-\mathrm{m}(\tau, \tau)}{t}}
\end{gathered}
$$

implies that

$$
\mathrm{F}_{\mathrm{m}}(\tau, \eta, \mathrm{t}) * M_{\tau, \kappa, t} * M_{\kappa, \eta, t} \geq \mathrm{F}_{\mathrm{m}}(\tau, \kappa, \mathrm{t}) * \mathrm{~F}_{\mathrm{m}}(\kappa, \eta, \mathrm{t}) *
$$

Thus all the remaining cases can be proved easily in similar way.

Definition 3.2. Let $\left(\mathrm{X}, \mathrm{F}_{\mathrm{m}}, *\right)$ be a non-Archimedean fuzzy m-metric space then

(1) A sequence $\left\{\vartheta_{n}\right\}$ in $\left(\mathrm{X}, \mathrm{F}_{\mathrm{m}}, *\right)$ is said to be convergent to $\vartheta \in \mathrm{X}$ iff for each $\varepsilon>0$, there exists a positive integer $\mathrm{k}>0$ such that $\mathrm{F}_{\mathrm{m}}\left(\vartheta_{n}, \vartheta, \mathrm{t}\right) \geq \mathrm{M}_{\vartheta_{n}, \vartheta, t}-\varepsilon$ fo all $\mathrm{n} \geq \mathrm{k}$.

That is, $\lim _{n \rightarrow \infty}\left\{\mathrm{F}_{\mathrm{m}}\left(\vartheta_{n}, \vartheta, \mathrm{t}\right)-\mathrm{M}_{\vartheta_{n}, \vartheta, t}\right\}=0$

(2) The sequence $\left\{\vartheta_{n}\right\}$ in $\left(\mathrm{X}, \mathrm{F}_{\mathrm{m}}, *\right)$ is said to be Cauchy if

$\lim _{n}\left\{\mathrm{~F}_{\mathrm{m}}\left(\vartheta_{n}, \vartheta_{m}, \mathrm{t}\right)-\mathrm{M}_{\vartheta_{n}, \vartheta_{m}, t}\right\}=0$ and $\lim _{m \rightarrow m}^{n, m}\left\{M_{\vartheta_{n}, \vartheta_{m}, t}-\mathrm{m}_{\vartheta_{n}, \vartheta_{m}, t}\right\}=0$ where $\mathrm{m}_{\vartheta_{n}, \vartheta_{m}, t}=\min \left\{\mathrm{F}_{\mathrm{m}}\right.$ $\left.\left(\vartheta_{n}^{n \rightarrow \vartheta_{n}}, t\right), \mathrm{F}_{\mathrm{m}}\left(\vartheta_{m}, \vartheta_{m}, t\right)\right\}$

(3) $\left(X, F_{m}, *\right)$ is said to be complete if every Cauchy sequence $\left\{\vartheta_{n}\right\}$ in $\mathrm{X}$ converges to a point $\vartheta$.

(4) Mapping $\mathrm{f}: \mathrm{X} \rightarrow \mathrm{X}$ is said to be continuous at $\vartheta \in \mathrm{X}$, if for each $\varepsilon>0$ there exists a $\delta>0$ such that whenever $\mathrm{F}_{\mathrm{m}}(\vartheta, \rho, \mathrm{t})>M_{\vartheta, \rho, t}-\delta$ implies $\mathrm{F}_{\mathrm{m}}(\mathrm{f} \vartheta, \mathrm{f} \rho, \mathrm{t})>M_{f \vartheta, f \rho, t}-$ $\varepsilon$ for all $\rho \in \mathrm{X}$. In terms of sequence whenever $\vartheta_{n} \rightarrow \vartheta$ then $\mathrm{f} \vartheta_{n} \rightarrow \mathrm{f} \vartheta$ as $\mathrm{n} \rightarrow \infty$.

That is, if $\lim _{n \rightarrow \infty}\left\{F_{\mathrm{m}}\left(\vartheta_{n}, \vartheta, \mathrm{t}\right)-\mathrm{M}_{\vartheta_{n}, \vartheta, \mathrm{t}}\right\}=0$ then $\lim _{n \rightarrow \infty}$ $\left\{\mathrm{F}_{\mathrm{m}}\left(\mathrm{f} \vartheta_{n}, \mathfrak{f \vartheta} \vartheta^{n}, \overrightarrow{\mathrm{t}}^{\infty}-\mathrm{M}_{\mathrm{f} \vartheta_{n}, \mathrm{f} \vartheta \mathrm{t}}\right\}=0\right.$

(5) $F_{m}$ is said to be upper semi-continuous on $X$ if for every $\vartheta \in \mathrm{X}$,

$\lim _{n \rightarrow \infty} \sup \mathrm{F}_{\mathrm{m}}\left(\vartheta_{n}, \vartheta, \mathrm{t}\right) \leq \mathrm{F}_{\mathrm{m}}(\vartheta, \mathrm{p}, \mathrm{t})$ whenever a sequence $\left\{\vartheta_{n}^{n \rightarrow \infty}\right\}$ in $\mathrm{X}$ which converges to a point $\mathrm{p} \in \mathrm{X}$.

(6) fuzzy m-metric space $\left(\mathrm{X}, \mathrm{F}_{\mathrm{m}}, *\right)$ is compact if every sequence in $\mathrm{X}$ has a convergent subsequence.

Theorem 3.1. A non-Archimedean Fuzzy m-metric $F_{m}$ $(\tau, \eta, \mathrm{t})$ is non-decreasing with respect to 't' for each $\tau$, $\eta \in \mathrm{X}$ and $\mathrm{t}>0$, if the continuous t-norm satisfies the following condition for all a,b,d $\in[0,1]$, 
$\mathrm{a} * \mathrm{~b} \geq \mathrm{a} * \mathrm{~d} \Rightarrow \mathrm{b} \geq \mathrm{d}$ for all a,b,d $\in[0,1]$

Proof. From (FMM 4) for all , $\eta, \kappa \in \mathrm{X}$ and t,s $>0$, we get

$$
\begin{gathered}
\mathrm{F}_{\mathrm{m}}(\tau, \eta, \max \{\mathrm{t}, \mathrm{s}\}) * M_{\tau, \kappa, t, s} * M_{\kappa, \eta, t, s} \geq \mathrm{F}_{\mathrm{m}}(\tau, \kappa, \mathrm{s}) * \\
\mathrm{~F}_{\mathrm{m}}(\kappa, \eta, \mathrm{t}) * M_{\tau, \eta, t, s} .
\end{gathered}
$$

Let $\mathrm{t}>\mathrm{s}$, and taking $\kappa=\eta$

$\mathrm{F}_{\mathrm{m}}(\tau, \eta, \mathrm{t}) * M_{\tau, \eta, t} * M_{\eta, \eta, t} \geq \mathrm{F}_{\mathrm{m}}(\tau, \eta, \mathrm{s}) * \mathrm{~F}_{\mathrm{m}}(\eta, \eta, \mathrm{t}) *$ $M_{\tau, \eta, t}$

or $\mathrm{F}_{\mathrm{m}}(\tau, \eta, \mathrm{t}) * M_{\tau, \eta, t} * \mathrm{~F}_{\mathrm{m}}(\eta, \eta, \mathrm{t}) \geq \mathrm{F}_{\mathrm{m}}(\tau, \eta, \mathrm{s}) * \mathrm{~F}_{\mathrm{m}}(\eta$, $\eta, \mathrm{t}) * M_{\tau, \eta, t}$

implies that $\mathrm{F}_{\mathrm{m}}(\tau, \eta, \mathrm{t}) \geq \mathrm{F}_{\mathrm{m}}(\tau, \eta$, s) by (1).

Theorem 3.2. Every partial fuzzy metric is a non-Archimedean fuzzy m-metric.

Proof: Let $(\mathrm{X}, \mathrm{P}, *)$ be a partial fuzzy metric space. It can be easily shown that the partial fuzzy metric satisfies the conditions (FMM 1), (FMM 3) and (FMM 5) of non-Archimedean fuzzy m-metric space (definition 3.1). Next, we have to prove the conditions (FMM 2) and (FMM 4).

(FMM 2) In PFMS, $\mathrm{P}(\tau, \tau, \mathrm{t}) \geq \mathrm{P}(\tau, \eta, \mathrm{t})$ for all $\tau \in \mathrm{X}$ implies that $\max \{\mathrm{P}(\tau, \tau, \mathrm{t}), \mathrm{P}(\eta, \eta, \mathrm{t})\} \geq \mathrm{P}(\tau, \eta, \mathrm{t})$ that is, (FMM 2) holds.

(FMM 4) Let $\mathrm{t}>\mathrm{s}$ and $\mathrm{P}$ be a partial fuzzy metric then for all $\tau, \eta, \kappa \in \mathrm{X}$, we have to show that

$$
\mathrm{P}(\tau, \eta, \mathrm{t}) * M_{\tau, \kappa, t} * M_{\kappa, \eta, t} \geq \mathrm{P}(\tau, \kappa, \mathrm{t}) * \mathrm{P}(\kappa, \eta, \mathrm{t}) *
$$

To prove above inequality, it is enough to take the following cases:

1. $\mathrm{P}(\tau, \tau, \mathrm{t})=\mathrm{P}(\eta, \eta, \mathrm{t})=\mathrm{P}(\kappa, \kappa, \mathrm{t})$

2. $\mathrm{P}(\tau, \tau, \mathrm{t})<\mathrm{P}(\eta, \eta, \mathrm{t})<\mathrm{P}(\kappa, \kappa, \mathrm{t})$

3. $\mathrm{P}(\tau, \tau, \mathrm{t})=\mathrm{P}(\eta, \eta, \mathrm{t})<\mathrm{P}(\kappa, \kappa, \mathrm{t})$

4. $\mathrm{P}(\tau, \tau, \mathrm{t})=\mathrm{P}(\eta, \eta, \mathrm{t})>\mathrm{P}(\kappa, \kappa, \mathrm{t})$

5. $\mathrm{P}(\tau, \tau, \mathrm{t})<\mathrm{P}(\eta, \eta, \mathrm{t})=\mathrm{P}(\kappa, \kappa, \mathrm{t})$

6. $\mathrm{P}(\tau, \tau, \mathrm{t})>\mathrm{P}(\eta, \eta, \mathrm{t})=\mathrm{P}(\kappa, \kappa, \mathrm{t})$

Without loss of generality, we take the following cases:

Case 4 : Since $\mathrm{P}(\tau, \eta, \mathrm{t}) * \mathrm{P}(\kappa, \kappa, \mathrm{t}) \geq \mathrm{P}(\tau, \kappa, \mathrm{t}) * \mathrm{P}(\kappa, \eta, \mathrm{t})$ as $\mathrm{P}(\tau, \tau, \mathrm{t})>\mathrm{P}(\kappa, \kappa, \mathrm{t})$

$\Rightarrow \mathrm{P}(\tau, \eta, \mathrm{t}) * \mathrm{P}(\tau, \tau, \mathrm{t}) \geq \mathrm{P}(\tau, \eta, \mathrm{t}) * \mathrm{P}(\kappa, \kappa, \mathrm{t}) \geq \mathrm{P}(\tau, \kappa, \mathrm{t})$

$* \mathrm{P}(\kappa, \eta, \mathrm{t})$

$\Rightarrow \mathrm{P}(\tau, \eta, \mathrm{t}) * \mathrm{P}(\tau, \tau, \mathrm{t}) * \mathrm{P}(\eta, \eta, \mathrm{t}) \geq \mathrm{P}(\tau, \kappa, \mathrm{t}) * \mathrm{P}(\kappa$,

$\eta, \mathrm{t}) * \mathrm{P}(\eta, \eta, \mathrm{t})$

$\Rightarrow \mathrm{P}(\tau, \eta, \mathrm{t}) * M_{\tau, \kappa, t} * M_{\kappa, \eta, t} \geq \mathrm{P}(\tau, \kappa, \mathrm{t}) * \mathrm{P}(\kappa, \eta, \mathrm{t}) *$ $M_{\tau, \eta, t}$.

Case 5: Again, $\mathrm{P}(\tau, \eta, \mathrm{t}) * \mathrm{P}(\kappa, \kappa, \mathrm{t}) \geq \mathrm{P}(\tau, \kappa, \mathrm{t}) * \mathrm{P}(\kappa$, $\eta, \mathrm{t})$

$\Rightarrow \mathrm{P}(\tau, \eta, \mathrm{t}) * \mathrm{P}(\kappa, \kappa, \mathrm{t}) * \mathrm{P}(\eta, \eta, \mathrm{t}) \geq \mathrm{P}(\tau, \kappa, \mathrm{t}) * \mathrm{P}(\kappa, \eta, \mathrm{t})$

$* \mathrm{P}(\eta, \eta, \mathrm{t})$

$\Rightarrow \mathrm{P}(\tau, \eta, \mathrm{t}) * M_{\tau, \kappa, t} * M_{\kappa, \eta, t} \geq \mathrm{P}(\tau, \kappa, \mathrm{t}) * \mathrm{P}(\kappa, \eta, \mathrm{t}) *$ $M_{\tau, \eta, t}$.
Case 6: As $\mathrm{P}(\tau, \eta, \mathrm{t}) * \mathrm{P}(\kappa, \kappa, \mathrm{t}) \geq \mathrm{P}(\tau, \kappa, \mathrm{t}) * \mathrm{P}(\kappa, \eta, \mathrm{t})$ ( In PFMS)

$\Rightarrow \mathrm{P}(\tau, \eta, \mathrm{t}) * \mathrm{P}(\kappa, \kappa, \mathrm{t}) * \mathrm{P}(\tau, \tau, \mathrm{t}) \geq \mathrm{P}(\tau, \kappa, \mathrm{t}) * \mathrm{P}(\kappa, \eta, \mathrm{t})$

$* \mathrm{P}(\tau, \tau, \mathrm{t})$

$\Rightarrow \mathrm{P}(\tau, \eta, \mathrm{t}) * M_{\tau, \kappa, t} * M_{\kappa, \eta, t} \geq \mathrm{P}(\tau, \kappa, \mathrm{t}) * \mathrm{P}(\kappa, \eta, \mathrm{t}) *$ $M_{\tau, \eta, t}$.

Similarly, we can prove all the left cases.

Thus, partial fuzzy metric satisfies all the conditions of non-Archimedean fuzzy m-metric. Therefore every partial fuzzy metric space is non-Archimedean fuzzy m-metric space.

Remark: Fuzzy metric $\Rightarrow$ Partial fuzzy metric $\Rightarrow$ non-Archimedean Fuzzy m- metric but not conversely.

Corresponding to every fuzzy metric and non-Archimedean fuzzy m-metric, we can introduce another non-Archimedean fuzzy m-metric as:

Example 3.3. Let $(\mathrm{X}, \mathrm{F}, *),\left(\mathrm{X}, \mathrm{F}_{\mathrm{m}}, *\right)$ be a fuzzy metric space and non-Archimedean fuzzy m-metric space respectively. Then the mappings $\mathrm{M}_{\mathrm{f}_{\mathrm{i}}}: \mathrm{X} \times \mathrm{X} \times(0, \infty) \rightarrow$ $[0,1], i=1,2$ defined by

1. $\mathrm{M}_{\mathrm{f}_{1}}(\tau, \eta, \mathrm{t})=\mathrm{F}(\tau, \eta, \mathrm{t}) * \mathrm{~F}_{\mathrm{m}}(\tau, \eta, \mathrm{t})$

2. $\quad \mathrm{M}_{\mathrm{f}_{2}}(\tau, \eta, \mathrm{t})=\mathrm{F}(\tau, \eta, \mathrm{t}) *$ a where $0<\mathrm{a}<1$

are fuzzy m-metric on X.

Proof: (i) To prove $M_{f_{1}}$ is fuzzy m-metric, it is enough to show that $\mathrm{M}_{\mathrm{f}_{1}}$ satisfies the conditions (FMM 2) and (FMM 4) since it is easy to prove the conditions (FMM 1), (FMM 3) and (FMM 5) of NAFmMS.

(FMM2) As $\mathrm{F}_{\mathrm{m}}(\tau, \eta, \mathrm{t})$ is fuzzy m-metric.

Therefore, $\max \left\{\mathrm{F}_{\mathrm{m}}(\tau, \tau, \mathrm{t}), \mathrm{F}_{\mathrm{m}}(\eta, \eta, \mathrm{t})\right\} \geq \mathrm{F}_{\mathrm{m}}(\tau, \eta, \mathrm{t})$

There are two possibilities:

(I) If $\max \left\{\mathrm{F}_{\mathrm{m}}(\tau, \tau, \mathrm{t}), \mathrm{F}_{\mathrm{m}}(\eta, \eta, \mathrm{t})\right\}=\mathrm{F}_{\mathrm{m}}(\tau, \tau, \mathrm{t})$

then $\mathrm{F}(\tau, \tau, \mathrm{t}) * \mathrm{~F}_{\mathrm{m}}(\tau, \tau, \mathrm{t}) \geq \mathrm{F}(\tau, \eta, \mathrm{t}) * \mathrm{~F}_{\mathrm{m}}(\tau, \eta, \mathrm{t})$.

(II) If $\max \left\{\mathrm{F}_{\mathrm{m}}(\tau, \tau, \mathrm{t}), \mathrm{F}_{\mathrm{m}}(\eta, \eta, \mathrm{t})\right\}=\mathrm{F}_{\mathrm{m}}(\eta, \eta, \mathrm{t})$

then $\mathrm{F}(\eta, \eta, \mathrm{t}) * \mathrm{~F}_{\mathrm{m}}(\eta, \eta, \mathrm{t}) \geq \mathrm{F}(\tau, \eta, \mathrm{t}) * \mathrm{~F}_{\mathrm{m}}(\tau, \eta, \mathrm{t})$.

Therefore, $\max \left\{\mathrm{F}(\tau, \tau, \mathrm{t}) * \mathrm{~F}_{\mathrm{m}}(\tau, \tau, \mathrm{t}), \mathrm{F}(\eta, \eta, \mathrm{t}) * \mathrm{~F}_{\mathrm{m}}(\eta, \eta, \mathrm{t})\right\} \geq$
$\mathrm{F}(\tau, \eta, \mathrm{t}) * \mathrm{~F}_{\mathrm{m}}(\tau, \eta \mathrm{t})$

that is, $\max \left\{\mathrm{M}_{\mathrm{f}_{1}}(\tau, \tau, \mathrm{t}), \mathrm{M}_{\mathrm{f}_{1}}(\eta, \eta, \mathrm{t})\right\} \geq \mathrm{M}_{\mathrm{f}_{1}}(\tau, \eta, \mathrm{t})$

implies that (FMM 2) holds.

(FMM 4) Let $t>s$. As $F$ is fuzzy metric and $F_{m}$ is a non-Archimedean fuzzy m- metric, so we have

$$
\mathrm{F}(\tau, \eta, \mathrm{t}) \geq \mathrm{F}(\tau, \kappa, \mathrm{t}) * \mathrm{~F}(\kappa, \eta, \mathrm{t})
$$

and

$$
\mathrm{F}_{\mathrm{m}}(\tau, \eta, \mathrm{t}) * M_{\tau, \kappa, t} * M_{\kappa, \eta, t} \geq \mathrm{F}_{\mathrm{m}}(\tau, \kappa, \mathrm{t}) * \mathrm{~F}_{\mathrm{m}}(\kappa, \eta, \mathrm{t}) *
$$

Using the property, $\mathrm{a} \geq \mathrm{b}$ and $\mathrm{c} \geq \mathrm{d}$ implies $\mathrm{a} * \mathrm{c} \geq \mathrm{b} *$ $\mathrm{d}$, we have

$$
\begin{gathered}
\mathrm{F}(\tau, \eta, \mathrm{t}) * \mathrm{~F}_{\mathrm{m}}(\tau, \eta, \mathrm{t}) * M_{\tau, \kappa, t} * M_{\kappa, \eta, t} \geq \mathrm{F}_{\mathrm{m}}(\tau, \kappa, \mathrm{t}) * \mathrm{~F}_{\mathrm{m}} \\
(\kappa, \eta, \mathrm{t}) * M_{\tau, \eta, t} * \mathrm{~F}(\tau, \kappa, \mathrm{t}) * \mathrm{~F}(\kappa, \eta, \mathrm{t})
\end{gathered}
$$

that is, 
$\left\{\mathrm{F}(\tau, \eta, \mathrm{t}) * \mathrm{~F}_{\mathrm{m}}(\tau, \eta, \mathrm{t})\right\} * M_{\tau, \kappa, t} * M_{\kappa, \eta, t} \geq\left\{\mathrm{F}_{\mathrm{m}}(\tau, \kappa, \mathrm{t})\right.$ $* \mathrm{~F}(\tau, \kappa, \mathrm{t})\} *\left\{\mathrm{~F}(\kappa, \eta, \mathrm{t}) * \mathrm{~F}_{\mathrm{m}}(\kappa, \eta, \mathrm{t})\right\} * M_{\tau, \eta, t}$

and we can write

$$
M_{\tau, \eta, t}=\max \left\{\mathrm{F}_{\mathrm{m}}(\tau, \tau, \mathrm{t}), \mathrm{F}_{\mathrm{m}}(\eta, \eta \mathrm{t})\right\}
$$

$=\max \left\{\mathrm{F}(\tau, \tau, \mathrm{t}) * \mathrm{~F}_{\mathrm{m}}(\tau, \tau, \mathrm{t}), \mathrm{F}(\eta, \eta, \mathrm{t}) * \mathrm{~F}_{\mathrm{m}}(\eta, \eta, \mathrm{t})\right\}$.

Hence, $\mathrm{M}_{\mathrm{f}_{1}}(\tau, \eta, \mathrm{t}) * M_{\tau, \kappa, t} * M_{\kappa, \eta, t} \geq \mathrm{M}_{\mathrm{f}_{1}}(\tau, \kappa, \mathrm{t}) *$ $\mathrm{M}_{\mathrm{f}_{1}}(\kappa, \eta, \mathrm{t}) * M_{\tau, \eta, t}$.

(ii) Since $\mathrm{M}_{\mathrm{f}_{2}}(\tau, \eta, \mathrm{t})=\mathrm{F}(\tau, \eta, \mathrm{t}) * \mathrm{a}$ is a partial fuzzy metric and we have already proved in Theorem 3.2, every partial fuzzy metric is a non-Archimedean fuzzy m-metric.

Definition 3.3. Let $\mathrm{F}_{\mathrm{m}}$ be a non-Archimedean fuzzy m-metric on a set X. For each $\epsilon>0$ and $\tau \in \mathrm{X}$, then open ball in non-Archimedean fuzzy m- metric space around $\tau$ of radius $\epsilon$ is

$$
\begin{gathered}
\mathrm{B}_{\epsilon}^{\mathrm{M}}(\tau)=\left\{\eta \in \mathrm{X}: \mathrm{F}_{\mathrm{m}}(\tau, \eta, \mathrm{t}) * \mathrm{~F}_{\mathrm{m}}(\eta, \eta, \mathrm{t})>M_{\tau, \eta, t} * \mathrm{~F}_{\mathrm{m}}\right. \\
(\tau, \tau, \mathrm{t})-\epsilon\} .
\end{gathered}
$$

Clearly, $\mathrm{B}_{\epsilon}^{\mathrm{M}}(\tau)$ is non-empty since $\tau \in \mathrm{B}_{\epsilon}^{\mathrm{M}}(\tau)$.

Remark: If for some $\tau, \eta \in \mathrm{X}$,

$$
M_{\tau, \eta, t}=\mathrm{F}_{\mathrm{m}}(\eta, \eta, \mathrm{t}) \geq \mathrm{F}_{\mathrm{m}}(\tau, \eta, \mathrm{t}) \geq \mathrm{F}_{\mathrm{m}}(\tau, \tau, \mathrm{t}) .
$$

Then, we have

$\mathrm{F}_{\mathrm{m}}(\tau, \eta, \mathrm{t}) \geq \mathrm{F}_{\mathrm{m}}(\tau, \tau, \mathrm{t})$

$\Rightarrow \mathrm{F}_{\mathrm{m}}(\tau, \eta, \mathrm{t}) * \mathrm{~F}_{\mathrm{m}}(\eta, \eta, \mathrm{t}) \geq \mathrm{F}_{\mathrm{m}}(\tau, \tau, \mathrm{t}) * \mathrm{~F}_{\mathrm{m}}(\eta, \eta, \mathrm{t})$

$=M_{\tau, \eta, t} * \mathrm{~F}_{\mathrm{m}}(\tau, \tau, \mathrm{t})$

$>M_{\tau, \eta, t} * \mathrm{~F}_{\mathrm{m}}(\tau, \tau, \mathrm{t})-\epsilon$

implies that $\eta \in \mathrm{B}_{\epsilon}^{\mathrm{M}}(\tau)$ for each $0<\epsilon<1$.

Theorem 3.3. A non-Archimedean fuzzy m-metric space is $T_{0}$.

Proof: Let $\left(\mathrm{X}, \mathrm{F}_{\mathrm{m}}, *\right)$ be a fuzzy m-metric space. Take $\tau, \eta \in \mathrm{X}$ be any two distinct point.

To prove, it is $\mathrm{T}_{0}$ space, it is enough to find an $\epsilon>0$ such that $\eta \notin \mathrm{B}_{\epsilon}^{\mathrm{M}}(\tau)$.

Here two cases arise:

Case I: If $\mathrm{F}_{\mathrm{m}}(\tau, \tau, \mathrm{t})=\mathrm{F}_{\mathrm{m}}(\eta, \eta, \mathrm{t})$

then $M_{\tau, \eta, t} * \mathrm{~F}_{\mathrm{m}}(\tau, \tau, \mathrm{t})=\mathrm{F}_{\mathrm{m}}(\tau, \tau, \mathrm{t}) * \mathrm{~F}_{\mathrm{m}}(\tau, \tau, \mathrm{t})$

$$
>\mathrm{F}_{\mathrm{m}}(\tau, \eta, \mathrm{t}) * \mathrm{~F}_{\mathrm{m}}(\eta, \eta, \mathrm{t})
$$

Suppose that $\mathrm{F}_{\mathrm{m}}(\tau, \tau, \mathrm{t}) * \mathrm{~F}_{\mathrm{m}}(\tau, \tau, \mathrm{t})=\mathrm{r}_{1}$ and $\mathrm{F}_{\mathrm{m}}(\tau, \eta, \mathrm{t}) * \mathrm{~F}_{\mathrm{m}}(\eta, \eta, \mathrm{t})=\mathrm{r}_{2}$ and choose $\epsilon_{1}=\frac{r_{1}-r_{2}}{10}>0$ such that $\mathrm{r}_{1}>\mathrm{r}_{2}+\epsilon_{1}$

that is, $M_{\tau, \eta, t} * \mathrm{~F}_{\mathrm{m}}(\tau, \tau, \mathrm{t})=\mathrm{F}_{\mathrm{m}}(\tau, \tau, \mathrm{t}) * \mathrm{~F}_{\mathrm{m}}(\tau, \tau, \mathrm{t})$

$>\mathrm{F}_{\mathrm{m}}(\tau, \eta, \mathrm{t}) * \mathrm{~F}_{\mathrm{m}}(\eta, \eta, \mathrm{t})+\epsilon_{1}$.

Case II: If $\mathrm{F}_{\mathrm{m}}(\tau, \tau, \mathrm{t})>\mathrm{F}_{\mathrm{m}}(\eta, \eta, \mathrm{t})$

then $M_{\tau, \eta, t} * \mathrm{~F}_{\mathrm{m}}(\tau, \tau, \mathrm{t})=\mathrm{F}_{\mathrm{m}}(\tau, \tau, \mathrm{t}) * \mathrm{~F}_{\mathrm{m}}(\tau, \tau, \mathrm{t})$

$>\mathrm{F}_{\mathrm{m}}(\tau, \eta, \mathrm{t}) * \mathrm{~F}_{\mathrm{m}}(\eta, \eta, \mathrm{t})$

that is, Case II reach at the same position of Case I.

Thus, we can find an $0<\epsilon_{1}=\frac{r_{1}-r_{2}}{10}<1$ such that

$$
\begin{gathered}
M_{\tau, \eta, t} * \mathrm{~F}_{\mathrm{m}}(\tau, \tau, \mathrm{t})=\mathrm{F}_{\mathrm{m}}(\tau, \tau, \mathrm{t}) * \mathrm{~F}_{\mathrm{m}}(\tau, \tau, \mathrm{t}) \\
>\mathrm{F}_{\mathrm{m}}(\tau, \eta, \mathrm{t}) * \mathrm{~F}_{\mathrm{m}}(\eta, \eta, \mathrm{t})+\epsilon_{1} .
\end{gathered}
$$

That is,

$$
M_{\tau, \eta, t} * \mathrm{~F}_{\mathrm{m}}(\tau, \tau, \mathrm{t})-\epsilon_{1}>\mathrm{F}_{\mathrm{m}}(\tau, \eta, \mathrm{t}) * \mathrm{~F}_{\mathrm{m}}(\eta, \eta, \mathrm{t}) .
$$

Therefore, for $\epsilon_{1}=\frac{r_{1}-r_{2}}{10}, \quad \eta \notin \mathrm{B}_{\epsilon}^{\mathrm{M}}(\tau)$ which completes the proof.

Theorem 3.4. Let $\left(\mathrm{X}, \mathrm{F}_{\mathrm{m}}, *\right)$ be a non-Archimedean fuzzy m-metric space and suppose that

$\mathfrak{I}_{M}=\{\mathrm{A} \subseteq \mathrm{X}: \eta \in \mathrm{A} \Leftrightarrow \exists t>0$ and $0<r<1$ such that $\left.\mathrm{B}_{\mathrm{r}}^{\mathrm{M}}(\eta) \subset \mathrm{A}\right\}$

be a subset of $\mathrm{P}(\mathrm{X})$ where $\mathrm{P}(\mathrm{X})$ denote the power set of $\mathrm{X}$ then $\mathfrak{I}_{M}$ is a topology on $\mathrm{X}$.

Proof: Clearly, $\emptyset, \mathrm{X} \in \mathfrak{I}_{M}$. Let $\mathrm{O}_{1}, \mathrm{O}_{2}, \mathrm{O}_{3}, \mathrm{O}_{4} \ldots \ldots$ $\in \mathfrak{I}_{M}$

Take $\mathrm{O}=\mathrm{U}_{i}\left(\mathrm{O}_{\mathrm{i}}\right)$. Let a $\in \mathrm{O}$ be arbitrary element.

$\Rightarrow \mathrm{a} \in \mathrm{O}_{\mathrm{i}}$ for some $i$

implies that there exist $\mathrm{t}>0$ and $0<\mathrm{r}_{\mathrm{i}}<1$ such that $\mathrm{B}_{r_{i}}^{\mathrm{M}}$ (a) $\subset \mathrm{O}_{\mathrm{i}} \subseteq \mathrm{U}_{i}\left(\mathrm{O}_{\mathrm{i}}\right)$

that is, a $\in \mathrm{B}_{r_{i}}^{\mathrm{M}}(\mathrm{a}) \subseteq \mathrm{U}_{i}\left(\mathrm{O}_{\mathrm{i}}\right)$

Now, take $\mathrm{O}_{1}, \mathrm{O}_{2}, \mathrm{O}_{3}, \ldots \ldots, \mathrm{O}_{\mathrm{n}} \in \mathfrak{I}_{M}$. Assume that $\mathrm{J}=$ $\cap_{i=1}^{n}\left(\mathrm{O}_{\mathrm{i}}\right)$.

Let a $\in \mathrm{J}$ be arbitrary element.

$\Rightarrow \mathrm{a} \in \mathrm{O}_{\mathrm{i}}$ for all $i=1,2, \ldots, \mathrm{n}$.

Then by definition of $\mathfrak{I}_{M}$ there exist $0<\mathrm{r}_{\mathrm{i}}<1$ and $\mathrm{t}_{\mathrm{i}}>0$ such that $\mathrm{B}_{r_{i}}^{\mathrm{M}}$ (a) $\subset \mathrm{O}_{\mathrm{i}}$ for all $i=1,2, \ldots, \mathrm{n}$.

Choose, $\mathrm{r}=\min _{i \in\{1,2, \ldots, n\}}\left\{r_{i}\right\}$ and

$\mathrm{t}=\max _{i \in\{1,2, \ldots, n\}}\left\{t_{i}\right\}$.

So, $\mathrm{r}<\mathrm{r}_{\mathrm{i}}$ for each $\mathrm{i}$ and it implies that if $\eta \in \mathrm{B}_{\mathrm{r}}^{\mathrm{M}}$ (a) then

$\mathrm{F}_{\mathrm{m}}(\mathrm{a}, \eta, \mathrm{t}) * \mathrm{~F}_{\mathrm{m}}(\eta, \eta, \mathrm{t})>M_{a, \eta, t} * \mathrm{~F}_{\mathrm{m}}(\mathrm{a}, \mathrm{a}, \mathrm{t})-\mathrm{r} \geq$ $M_{a, \eta, t} * \mathrm{~F}_{\mathrm{m}}(\mathrm{a}, \mathrm{a}, \mathrm{t})-r_{i}$ for each $i$ and $\mathrm{t}>0$.

$\Rightarrow \eta \in \mathrm{B}_{r_{i}}^{\mathrm{M}}$ (a) $\subset \mathrm{O}_{\mathrm{i}}$ for all $i=1,2, \ldots, \mathrm{n}$

$\Rightarrow \mathrm{B}_{\mathrm{r}}^{\mathrm{M}}(\mathrm{a}) \subset \mathrm{B}_{r_{i}}^{\mathrm{M}}(\mathrm{a}) \subset \mathrm{O}_{\mathrm{i}}$ for each $\mathrm{i}$

$\Rightarrow \mathrm{a} \in \mathrm{B}_{\mathrm{r}}^{\mathrm{M}}$ (a) $\subset \cap_{i=1}^{n}\left(\mathrm{O}_{\mathrm{i}}\right) \in \widetilde{I}_{M}$.

Hence, $\mathfrak{J}_{M}$ is a topology on $\mathrm{X}$.

Lemma 3.1. Let $\left(\mathrm{X}, \mathrm{F}_{\mathrm{m}}, *\right)$ be a non-Archimedean fuzzy $\mathrm{m}$-metric space with $\mathrm{a} * \mathrm{~b}=\mathrm{ab}$ for all $\mathrm{a}, \mathrm{b} \in[0,1]$. Define $\mathrm{a}$ mapping $\mathrm{m}: \mathrm{X} \times \mathrm{X} \rightarrow[0, \infty)$ such that

$$
\mathrm{m}(\tau, \eta)=\sup _{\alpha \in(0,1)} \int_{\alpha}^{1} \log _{\mathrm{a}}\left(\mathrm{F}_{\mathrm{m}}(\tau, \eta, \mathrm{t})\right) \mathrm{dt}, 0<\mathrm{a}<1
$$

then $\mathrm{m}$ is $\mathrm{m}$ - metric on $\mathrm{X}$ for fixed $0<\alpha<1$.

Proof. Clearly, $\mathrm{m}(\tau, \eta)$ is well defined. For all $0<\mathrm{t}<1$

1. $\mathrm{m}(\tau, \tau)=\mathrm{m}(\tau, \eta)=\mathrm{m}(\eta, \eta)$ if and only if $\mathrm{F}_{\mathrm{m}}(\tau, \tau, \mathrm{t})=$ $\mathrm{F}_{\mathrm{m}}(\tau, \eta, \mathrm{t})=\mathrm{F}_{\mathrm{m}}(\eta, \eta, \mathrm{t})$

2. If $\max \left\{\mathrm{F}_{\mathrm{m}}(\tau, \tau, \mathrm{t}), \mathrm{F}_{\mathrm{m}}(\eta, \eta, \mathrm{t})\right\}=\mathrm{F}_{\mathrm{m}}(\tau, \tau, \mathrm{t}) \geq \mathrm{F}_{\mathrm{m}}(\tau$, $\eta, \mathrm{t})$

then $\mathrm{m}(\tau, \tau)=\sup _{\alpha \in(0,1)} \int_{\alpha}^{1} \log _{\mathrm{a}}\left(\mathrm{F}_{\mathrm{m}}(\tau, \tau, \mathrm{t})\right)$

$\leq \sup _{\alpha \in(0,1)} \int_{\alpha}^{1} \log _{\mathrm{a}}\left(\mathrm{F}_{\mathrm{m}}(\tau, \eta, \mathrm{t})\right)=\mathrm{m}(\tau, \eta)\left(\right.$ since $\log _{\mathrm{a}}$ is decreasing)

$\Rightarrow m_{\tau, \eta} \leq \mathrm{m}(\tau, \eta)$ where $m_{\tau, \eta}=\min \{\mathrm{m}(\tau, \tau), \mathrm{m}(\eta, \eta)\}$ and if $\max \left\{\mathrm{F}_{\mathrm{m}}(\tau, \tau, \mathrm{t}), \mathrm{F}_{\mathrm{m}}(\eta, \eta, \mathrm{t})\right\}=\mathrm{F}_{\mathrm{m}}(\eta, \eta, \mathrm{t}) \geq$ $\mathrm{F}_{\mathrm{m}}(\tau, \eta, \mathrm{t})$.

Similarly as we computed above, we obtain

$$
\mathrm{m}_{\tau, \eta} \leq \mathrm{m}(\tau, \eta) .
$$


3. $\mathrm{F}_{\mathrm{m}}(\tau, \eta, \mathrm{t})=\mathrm{F}_{\mathrm{m}}(\eta, \tau, \mathrm{t})$ implies $\mathrm{m}(\tau, \eta)=\mathrm{m}(\eta, \tau)$ for all $\tau, \eta \in \mathrm{X}$.

4. Since $\mathrm{F}_{\mathrm{m}}(\tau, \eta, \mathrm{t}) * M_{\tau, \kappa, t} * M_{\kappa, \eta, t} \geq \mathrm{F}_{\mathrm{m}}(\tau, \kappa, \mathrm{t})$ $* \mathrm{~F}_{\mathrm{m}}(\kappa, \eta, \mathrm{t}) * M_{\tau, \eta, t}$

that is, $\mathrm{F}_{\mathrm{m}}(\tau, \eta, \mathrm{t}) \cdot M_{\tau, \kappa, t} \cdot M_{\kappa, \eta, t} \geq \mathrm{F}_{\mathrm{m}}(\tau, \kappa, \mathrm{t}) \cdot \mathrm{F}_{\mathrm{m}}$ $(\kappa, \eta, \mathrm{t}) \cdot M_{\tau, \eta, t}$

and $\log _{\mathrm{a}}$ is decreasing, so we have

$$
\begin{aligned}
& \sup _{\alpha \in(0,1)} \int_{\alpha}^{1} \log _{\mathrm{a}}\left(\mathrm{F}_{\mathrm{m}}(\tau, \eta, \mathrm{t})\right) d t \\
& +\sup _{\alpha \in(0,1)} \int_{\alpha}^{1} \log _{\mathrm{a}}\left(M_{\tau, \kappa, t}\right) d t \\
& +\sup _{\alpha \in(0,1)} \int_{\alpha}^{1} \log _{\mathrm{a}}\left(M_{\kappa, \eta, t}\right) d t \\
& \leq \sup _{\alpha \in(0,1)} \int_{\alpha}^{1} \log _{\mathrm{a}}\left(\mathrm{F}_{\mathrm{m}}(\tau, \kappa, \mathrm{t})\right) \mathrm{dt}+ \\
& \sup _{\alpha \in(0,1)} \int_{\alpha}^{1} \log _{\mathrm{a}}\left(\mathrm{F}_{\mathrm{m}}(\kappa, \eta, \mathrm{t})\right) \mathrm{dt}+ \\
& \sup _{\alpha \in(0,1)} \int_{\alpha}^{1} \log _{\mathrm{a}}\left(M_{\tau, \eta, t}\right) \mathrm{dt} .
\end{aligned}
$$

To prove (4), it is enough to take the following cases:

(i) $\mathrm{F}_{\mathrm{m}}(\tau, \tau, \mathrm{t})=\mathrm{F}_{\mathrm{m}}(\eta, \eta, \mathrm{t})=\mathrm{F}_{\mathrm{m}}(\kappa, \kappa, \mathrm{t})$

(ii) $\mathrm{F}_{\mathrm{m}}(\tau, \tau, \mathrm{t})<\mathrm{F}_{\mathrm{m}}(\eta, \eta, \mathrm{t})<\mathrm{F}_{\mathrm{m}}(\kappa, \kappa, \mathrm{t})$

(iii) $\mathrm{F}_{\mathrm{m}}(\tau, \tau, \mathrm{t})=\mathrm{F}_{\mathrm{m}}(\eta, \eta, \mathrm{t})<\mathrm{F}_{\mathrm{m}}(\kappa, \kappa, \mathrm{t})$

(iv) $\mathrm{F}_{\mathrm{m}}(\tau, \tau, \mathrm{t})=\mathrm{F}_{\mathrm{m}}(\eta, \eta, \mathrm{t})>\mathrm{F}_{\mathrm{m}}(\kappa, \kappa, \mathrm{t})$

(v) $\mathrm{F}_{\mathrm{m}}(\tau, \tau, \mathrm{t})<\mathrm{F}_{\mathrm{m}}(\eta, \eta, \mathrm{t})=\mathrm{F}_{\mathrm{m}}(\kappa, \kappa, \mathrm{t})$

(vi) $\mathrm{F}_{\mathrm{m}}(\tau, \tau, \mathrm{t})>\mathrm{F}_{\mathrm{m}}(\eta, \eta, \mathrm{t})=\mathrm{F}_{\mathrm{m}}(\kappa, \kappa, \mathrm{t})$.

If we consider the (i) case

That is, $\mathrm{F}_{\mathrm{m}}(\tau, \tau, \mathrm{t})=\mathrm{F}_{\mathrm{m}}(\eta, \eta, \mathrm{t})=\mathrm{F}_{\mathrm{m}}(\kappa, \kappa, \mathrm{t})$

$$
\text { then } \mathrm{m}(\tau, \tau)=\mathrm{m}(\eta, \eta)=\mathrm{m}(\kappa, \kappa)
$$

and from (2), we have

$$
\begin{gathered}
\mathrm{m}(\tau, \eta)+m_{\tau, \kappa}+m_{\eta, \kappa} \leq \mathrm{m}(\tau, \kappa)+\mathrm{m}(\kappa, \eta)+m_{\tau, \eta} \\
\quad \text { (using(3)). }
\end{gathered}
$$

If we consider the (ii) case

that is, $\mathrm{F}_{\mathrm{m}}(\tau, \tau, \mathrm{t})<\mathrm{F}_{\mathrm{m}}(\eta, \eta, \mathrm{t})<\mathrm{F}_{\mathrm{m}}(\kappa, \kappa, \mathrm{t})$

then $\mathrm{m}(\tau, \tau)>\mathrm{m}(\eta, \eta)>\mathrm{m}(\kappa, \kappa)$ since $\log _{\mathrm{a}}$ is a decre asing function

and from (2), we have

$\mathrm{m}(\tau, \eta)+\mathrm{m}(\kappa, \kappa)+\mathrm{m}(\kappa, \kappa) \leq \mathrm{m}(\tau, \kappa)+\mathrm{m}(\kappa, \eta)+\mathrm{m}(\eta, \eta)$ implies that

$\mathrm{m}(\tau, \eta)+m_{\tau, \kappa}+m_{\eta, \kappa} \leq \mathrm{m}(\tau, \kappa)+\mathrm{m}(\kappa, \eta)+m_{\tau, \eta}$ (using $(4))$.

Similarly, in left cases we can easily show that

$$
\mathrm{m}(\tau, \eta)+m_{\tau, \kappa}+m_{\eta, \kappa} \leq \mathrm{m}(\tau, \kappa)+\mathrm{m}(\kappa, \eta)+m_{\tau, \eta} .
$$

Thus, $\mathrm{m}$ is a m-metric on $\mathrm{X}$.

Lemma 3.2. Let $\left\{\vartheta_{n}\right\}$ be a convergent sequence in non-Archimedean fuzzy m-metric space $\left(\mathrm{X}, \mathrm{F}_{\mathrm{m}}, *\right)$ with property $\mathrm{a} * \mathrm{~b} \geq \mathrm{a} * \mathrm{c} \Rightarrow \mathrm{b} \geq \mathrm{c}$ for all $\mathrm{a}, \mathrm{b}, c \in[0,1]$. If $\vartheta_{n} \rightarrow \vartheta$ and $\vartheta_{n} \rightarrow \rho$ then
$\mathrm{F}_{\mathrm{m}}(\vartheta, \rho, \mathrm{t})=\max \left\{\mathrm{F}_{\mathrm{m}}(\vartheta, \vartheta, \mathrm{t}), \mathrm{F}_{\mathrm{m}}(\rho, \rho, \mathrm{t})\right\}$.

Further, if $\left.\mathrm{F}_{\mathrm{m}}(\vartheta, \vartheta, \mathrm{t})=\mathrm{F}_{\mathrm{m}}(\rho, \rho, \mathrm{t})\right\}$ then $\vartheta=\rho$.

Proof: By (FMM 6), we have

$\mathrm{F}_{\mathrm{m}}(\vartheta, \rho, \mathrm{t}) * \mathrm{M}_{\vartheta, \vartheta_{\mathrm{n}}, t} * \mathrm{M}_{\vartheta_{\mathrm{n}}, \rho, \mathrm{t}} \geq \mathrm{F}_{\mathrm{m}}\left(\mathrm{x}, \vartheta_{n}, \mathrm{t}\right) * \mathrm{~F}_{\mathrm{m}}\left(\vartheta_{n}, \rho, \mathrm{t}\right)$

$* \mathrm{M}_{\vartheta, \rho, \mathrm{t}}$.

As $\vartheta_{n} \rightarrow \vartheta$ and $\vartheta_{n} \rightarrow \rho$ then $\lim _{n \rightarrow \infty} \mathrm{F}_{\mathrm{m}}\left(\vartheta_{n}, \vartheta, \mathrm{t}\right)$ $=\lim _{n \rightarrow \infty} \mathrm{M}_{\vartheta_{n}, \vartheta, t}$ and $\lim _{n \rightarrow \infty} \mathrm{F}_{\mathrm{m}}\left(\vartheta_{n}, \rho, \mathrm{t}\right)=\lim _{n \rightarrow \infty} \mathrm{M}_{\vartheta_{n}, \rho, t}$.

Since $*$ is continuous t-norm and by property $\mathrm{a} * \mathrm{~b} \geq$ $\mathrm{a} * \mathrm{c} \Rightarrow \mathrm{b} \geq \mathrm{c}$, taking limit $\mathrm{n} \rightarrow \infty$ in (5), we have $\mathrm{F}_{\mathrm{m}}$ $(\vartheta, \rho, \mathrm{t}) \geq \mathrm{M}_{\vartheta, \rho, \mathrm{t}}$. But $\mathrm{M}_{\vartheta, \rho, \mathrm{t}} \geq \mathrm{F}_{\mathrm{m}}(\vartheta, \rho, \mathrm{t})$ implies that $\mathrm{F}_{\mathrm{m}}(\vartheta, \rho, \mathrm{t})=\mathrm{M}_{\vartheta, \rho, \mathrm{t}}$. Hence, by assumption we have $\vartheta=$ $\rho$.

Lemma 3.3. Let $\left\{\vartheta_{\mathrm{n}}\right\}$ and $\left\{\sigma_{\mathrm{n}}\right\}$ be two sequence in non-Archimedean fuzzy m-metric space $\left(\mathrm{X}, \mathrm{F}_{\mathrm{m}}, *\right)$ converges to $\vartheta$ and $\sigma$ respectively. In addition, assume that

$\lim _{n \rightarrow \infty} \max \left\{\mathrm{F}_{\mathrm{m}}\left(\vartheta_{\mathrm{n}}, \vartheta_{\mathrm{n}}, \mathrm{t}\right), \mathrm{F}_{\mathrm{m}}\left(\sigma_{\mathrm{n}}, \sigma_{\mathrm{n}}, \mathrm{t}\right)\right\}=\max \left\{\mathrm{F}_{\mathrm{m}}(\vartheta, \vartheta\right.$, $\left.\mathrm{t}), \mathrm{F}_{\mathrm{m}}(\sigma, \sigma, \mathrm{t})\right\}$

with $\mathrm{a} * \mathrm{~b} \geq \mathrm{a} * \mathrm{c} \Rightarrow \mathrm{b} \geq \mathrm{c}$ for all $\mathrm{a}, \mathrm{b}, \mathrm{c} \in[0,1]$.

Then $\lim _{n \rightarrow \infty} \mathrm{F}_{\mathrm{m}}\left(\vartheta_{\mathrm{n}}, \sigma_{\mathrm{n}}, \mathrm{t}\right)=\mathrm{F}_{\mathrm{m}}(\vartheta, \sigma, \mathrm{t})$. In particular,

$\lim _{n \rightarrow \infty} \mathrm{F}_{\mathrm{m}}\left(\vartheta_{\mathrm{n}}, \vartheta_{\mathrm{n}+1}, \mathrm{t}\right)=\mathrm{F}_{\mathrm{m}}(\vartheta, \vartheta, \mathrm{t})$ if $\lim _{n \rightarrow \infty} \max \left\{\mathrm{F}_{\mathrm{m}}\left(\vartheta_{\mathrm{n}}, \vartheta_{\mathrm{n}}\right.\right.$, t), $\left.F_{m}\left(\vartheta_{n+1}, \vartheta_{n+1}, t\right)\right\}=F_{m}(\vartheta, \vartheta, t)$.

Proof: By (FMM 6),

$\mathrm{F}_{\mathrm{m}}\left(\vartheta_{\mathrm{n}}, \sigma_{\mathrm{n}}, \mathrm{t}\right) * \mathrm{M}_{\vartheta_{n}, \vartheta, t} * \mathrm{M}_{\sigma_{n}, \vartheta, t} \geq \mathrm{F}_{\mathrm{m}}\left(\vartheta_{\mathrm{n}}, \vartheta, \mathrm{t}\right) * \mathrm{~F}_{\mathrm{m}}(\vartheta$, $\left.\sigma_{\mathrm{n}}, \mathrm{t}\right) * \mathrm{M}_{\vartheta_{n}, \sigma_{n}, t}$.

$\mathrm{F}_{\mathrm{m}}\left(\vartheta_{\mathrm{n}}, \sigma_{\mathrm{n}}, \mathrm{t}\right) * \mathrm{M}_{\vartheta_{n, \vartheta}, t} * M_{\sigma_{n}, \vartheta, t} * \mathrm{M}_{\sigma_{n}, \sigma, t} \geq \mathrm{F}_{\mathrm{m}}\left(\vartheta_{\mathrm{n}}, \vartheta, \mathrm{t}\right)$

$* \mathrm{~F}_{\mathrm{m}}\left(\vartheta, \sigma_{\mathrm{n}}, \mathrm{t}\right) * \mathrm{M}_{\vartheta_{n}, \sigma_{n}, t} * \mathrm{M}_{\sigma_{n}, \sigma, t}$.

Now, $\mathrm{F}_{\mathrm{m}}\left(\vartheta, \sigma_{\mathrm{n}}, \mathrm{t}\right) * \mathrm{M}_{\vartheta, \sigma, \mathrm{t}} * \mathrm{M}_{\sigma_{n}, \sigma, t} \geq \mathrm{F}_{\mathrm{m}}(\vartheta, \sigma, \mathrm{t}) * \mathrm{~F}_{\mathrm{m}}$ $\left(\sigma, \sigma_{\mathrm{n}}, \mathrm{t}\right) * \mathrm{M}_{\sigma_{n}, \vartheta, t}$.

Now taking lim sup of (8) and (9), we have

$$
\begin{aligned}
& \lim _{n \rightarrow \infty} \sup \left\{\mathrm{F}_{\mathrm{m}}\left(\vartheta_{\mathrm{n}}, \sigma_{\mathrm{n}}, \mathrm{t}\right) * \mathrm{M}_{\vartheta_{n}, \vartheta, t} * \mathrm{M}_{\sigma_{n}, \vartheta, t} * \mathrm{M}_{\sigma_{n}, \sigma, t}\right\} \\
& \geq \lim _{n \rightarrow \infty} \sup \left\{\mathrm{F}_{\mathrm{m}}\left(\vartheta_{\mathrm{n}}, \vartheta, \mathrm{t}\right) * \mathrm{~F}_{\mathrm{m}}\left(\vartheta, \sigma_{\mathrm{n}}, \mathrm{t}\right) * \mathrm{M}_{\vartheta_{n}, \sigma_{n}, t} * \mathrm{M}_{\sigma_{n}, \sigma, t}\right\} \\
& \geq \lim _{n \rightarrow \infty} \sup \mathrm{F}_{\mathrm{m}}\left(\vartheta_{\mathrm{n}}, \vartheta, \mathrm{t}\right) * \lim _{n \rightarrow \infty} \sup \left\{\mathrm{F}_{\mathrm{m}}\left(\vartheta, \sigma_{n}, \mathrm{t}\right) *\right. \\
&\left.\mathrm{M}_{\vartheta_{n}, \sigma_{n}, t} * \mathrm{M}_{\sigma_{n}, \sigma, t}\right\}
\end{aligned}
$$

and

$\lim _{n \rightarrow \infty} \sup \left\{\mathrm{F}_{\mathrm{m}}\left(\vartheta, \sigma_{\mathrm{n}}, \mathrm{t}\right) * \mathrm{M}_{\vartheta, \sigma, \mathrm{t}} * \mathrm{M}_{\sigma_{n}, \sigma, t}\right\} \geq \lim _{n \rightarrow \infty} \sup \left\{\mathrm{F}_{\mathrm{m}}(\vartheta\right.$,

$$
\left.\sigma, \mathrm{t}) * \mathrm{~F}_{\mathrm{m}}\left(\sigma, \sigma_{\mathrm{n}}, \mathrm{t}\right) * \mathrm{M}_{\sigma_{n}, \vartheta, t}\right\} .
$$

From (6), (7), (8a) and (9a), we can write

$$
\begin{gathered}
\quad \lim _{n \rightarrow \infty} \sup \left\{\mathrm{F}_{\mathrm{m}}\left(\vartheta_{\mathrm{n}}, \sigma_{\mathrm{n}}, \mathrm{t}\right) * \mathrm{M}_{\vartheta_{n}, \vartheta, t} * \mathrm{M}_{\sigma_{n}, \vartheta, t} * \mathrm{M}_{\sigma_{n}, \sigma, t}\right\} \\
\geq \lim _{n \rightarrow \infty} \sup \mathrm{F}_{\mathrm{m}}\left(\vartheta_{\mathrm{n}}, \vartheta, \mathrm{t}\right) * \lim _{n \rightarrow \infty} \sup \left\{\mathrm { F } _ { \mathrm { m } } ( \vartheta , \sigma , \mathrm { t } ) * \mathrm { F } _ { \mathrm { m } } \left(\sigma, \sigma_{\mathrm{n}},\right.\right. \\
\left.\mathrm{t}) * \mathrm{M}_{\sigma_{n}, \vartheta, t}\right\} \\
\geq \lim _{n \rightarrow \infty} \sup \left(\mathrm { F } _ { \mathrm { m } } ( \vartheta _ { \mathrm { n } } , \vartheta , \mathrm { t } ) * \mathrm { F } _ { \mathrm { m } } ( \vartheta , \sigma , \mathrm { t } ) * \operatorname { l i m } _ { n \rightarrow \infty } \left(\sup \mathrm{F}_{\mathrm{m}}(\sigma,\right.\right. \\
\left.\left.\sigma_{\mathrm{n}}, \mathrm{t}\right) * \mathrm{M}_{\sigma_{n}, \vartheta, t}\right) .
\end{gathered}
$$


Here, $\lim _{n \rightarrow \infty} \sup \left(\mathrm{F}_{\mathrm{m}}(\sigma, \sigma \mathrm{n}, \mathrm{t}) * \mathrm{M}_{\sigma_{n}, \vartheta, t}\right)=\lim _{n \rightarrow \infty} \sup$ $\left(\mathrm{M}_{\sigma_{n}, \vartheta, t} * \mathrm{M}_{\sigma_{n}, \sigma, t}\right)$ and $\lim _{n \rightarrow \infty} \mathrm{F}_{\mathrm{m}}(\vartheta \mathrm{n}, \vartheta, \mathrm{t})=\lim _{n \rightarrow \infty} \mathrm{M}_{\vartheta_{n}, \vartheta, t}$.

Therefore,

$$
\lim _{n \rightarrow \infty} \mathrm{F}_{\mathrm{m}}\left(\vartheta_{\mathrm{n}}, \sigma_{\mathrm{n}}, \mathrm{t}\right) \geq \mathrm{F}_{\mathrm{m}}(\vartheta, \sigma, \mathrm{t}) .
$$

Also,

$$
\begin{aligned}
\mathrm{F}_{\mathrm{m}}(\vartheta, \sigma, \mathrm{t}) * \mathrm{M}_{\vartheta_{n}, \vartheta, t} * \mathrm{M}_{\vartheta_{n}, \sigma, t} \geq \mathrm{F}_{\mathrm{m}}\left(\vartheta, \vartheta_{\mathrm{n}}, \mathrm{t}\right) * \mathrm{~F}_{\mathrm{m}}\left(\vartheta_{\mathrm{n}}, \sigma,\right. \\
\mathrm{t}) * \mathrm{M}_{\vartheta, \sigma, t}
\end{aligned}
$$

implies that

$$
\begin{aligned}
\mathrm{F}_{\mathrm{m}}(\vartheta, \sigma, \mathrm{t}) * \mathrm{M}_{\vartheta_{n}, \vartheta, t} * \mathrm{M}_{\vartheta_{n}, \sigma, t} * \mathrm{M}_{\sigma_{n}, \sigma, t} \geq \mathrm{F}_{\mathrm{m}}\left(\vartheta, \vartheta_{\mathrm{n}}, \mathrm{t}\right) \\
* \mathrm{~F}_{\mathrm{m}}\left(\vartheta_{\mathrm{n}}, \sigma, \mathrm{t}\right) * \mathrm{M}_{\vartheta, \sigma, t} * \mathrm{M}_{\sigma_{n}, \sigma, t .} \\
\text { Now, } \mathrm{F}_{\mathrm{m}}\left(\vartheta_{\mathrm{n}}, \sigma, \mathrm{t}\right) * \mathrm{M}_{\vartheta_{n}, \sigma_{n}, t} * \mathrm{M}_{\sigma_{n}, \sigma, t} \geq \mathrm{F}_{\mathrm{m}}\left(\vartheta_{\mathrm{n}}, \sigma_{\mathrm{n}}, \mathrm{t}\right) \\
* \mathrm{~F}_{\mathrm{m}}\left(\sigma_{\mathrm{n}}, \sigma, \mathrm{t}\right) * \mathrm{M}_{\vartheta_{n}, \sigma, t .}
\end{aligned}
$$

From (11), (12) and taking lim sup, it follows that

$$
\begin{gathered}
\lim _{n \rightarrow \infty} \sup \left\{\mathrm{F}_{\mathrm{m}}(\vartheta, \sigma, \mathrm{t}) * \mathrm{M}_{\vartheta_{n}, \vartheta, t} * \mathrm{M}_{\vartheta_{n}, \sigma, t} * \mathrm{M}_{\sigma_{n}, \sigma, t}\right\} \\
\geq \lim _{n \rightarrow \infty} \sup \left\{\mathrm { F } _ { \mathrm { m } } ( \vartheta , \vartheta _ { \mathrm { n } } , \mathrm { t } ) * \mathrm { F } _ { \mathrm { m } } ( \vartheta _ { \mathrm { n } } , \sigma _ { \mathrm { n } } , \mathrm { t } ) * \mathrm { F } _ { \mathrm { m } } \left(\sigma_{\mathrm{n}}, \sigma,\right.\right. \\
\left.\mathrm{t}) * \mathrm{M}_{\vartheta_{n}, \sigma, t}\right\} .
\end{gathered}
$$

Using the assumptions of sequences $\left\{\vartheta_{\mathrm{n}}\right\},\left\{\sigma_{\mathrm{n}}\right\}$ and (6), we have

$$
\mathrm{F}_{\mathrm{m}}(\vartheta, \sigma, \mathrm{t}) \geq \lim _{n \rightarrow \infty} \mathrm{F}_{\mathrm{m}}\left(\vartheta_{\mathrm{n}}, \sigma_{\mathrm{n}}, \mathrm{t}\right) .
$$

Thus from (10) and (13), we can write

$$
\lim _{n \rightarrow \infty} \mathrm{F}_{\mathrm{m}}\left(\vartheta_{\mathrm{n}}, \sigma_{\mathrm{n}}, \mathrm{t}\right)=\mathrm{F}_{\mathrm{m}}(\vartheta, \sigma, \mathrm{t}) .
$$

Hence the result.

Lemma 3.4 Let $\left\{\vartheta_{\mathrm{n}}\right\}$ be a sequence in non-Archimedean fuzzy $\mathrm{m}$-metric space $\left(\mathrm{X}, \mathrm{F}_{\mathrm{m}}, *\right)$ converges to $\vartheta$. In addition, assume that $\lim _{n \rightarrow \infty} \mathrm{F}_{\mathrm{m}}\left(\vartheta_{\mathrm{n}}, \vartheta_{\mathrm{n}}, \mathrm{t}\right)=\mathrm{F}_{\mathrm{m}}(\vartheta, \vartheta$, t $)$ with $\mathrm{a} * \mathrm{~b} \geq$ $\mathrm{a} * \mathrm{c} \Rightarrow \mathrm{b} \geq \mathrm{c}$ for all $\mathrm{a}, \mathrm{b} \in[0,1]$.

Then for every $z \in X, \lim _{n \rightarrow \infty} F_{m}\left(\vartheta_{n}, z, t\right)=F_{m}(\vartheta, z, t)$.

Proof. Proof of Lemma 3.4 follow from Lemma 3.3.

\section{Fixed Point Results}

In this part, we present some fixed point results in the context of non-Archimedean fuzzy m-metric space. Let $\Psi$ be a collection of functions $\psi:[0,1] \rightarrow[0,1]$ which satisfies the following properties:

(p1) $\psi$ is strictly decreasing and left continuous;

(p2) $\psi(\mu)=0$ if and only if $\mu=1$.

Theorem 4.1. Let $\left(\mathrm{X}, \mathrm{F}_{\mathrm{m}}, *\right)$ be a complete non-Archimedean fuzzy m-metric space with graph $\mathrm{G}=(\mathrm{V}, \mathrm{E})$ and with the property $\mathrm{a} * \mathrm{~b} \geq \mathrm{a} * \mathrm{c} \Rightarrow \mathrm{b} \geq \mathrm{c}$ for all $\mathrm{a}, \mathrm{b} \in[0,1]$. Suppose $\mathrm{T}$ be a self- mapping on $\mathrm{X}$ and $\mathrm{K}$ be $\mathrm{a}$ function from $(0, \infty)$ into $(0,1)$. If for any $t>0$, T satisfies the following condition:

$$
\psi\left(\mathrm{F}_{\mathrm{m}}(\mathrm{T} \vartheta, \mathrm{T} \sigma, \mathrm{t})\right) \leq \mathrm{K}(\mathrm{t}) \psi\left(\mathrm{F}_{\mathrm{m}}(\vartheta, \sigma, \mathrm{t})\right)
$$

where $\psi \in \Psi$ and $\vartheta, \sigma \in E(\tilde{G}), \vartheta \neq \sigma$ with the following properties:

(a) If a sequence $\vartheta_{n}=\mathrm{T} \vartheta_{n-1}$ then $\left(\vartheta_{n}, \vartheta_{m}\right) \in E(\tilde{G})$ for all $\mathrm{n}, \mathrm{m}$ and if this sequence $\left\{\vartheta_{n}\right\}$ converges to $\vartheta$ then there exist a subsequence $\left\{\vartheta_{k(n)}\right\}$ of $\left\{\vartheta_{n}\right\}$ such that $\left(\vartheta_{k(n)}, \vartheta\right) \in E(\tilde{G})$ for all $\mathrm{n}$;

(b) Assume that $\lim _{n \rightarrow \infty} \mathrm{F}_{\mathrm{m}}\left(\vartheta_{n}, \vartheta_{n}, \mathrm{t}\right)=\mathrm{F}_{\mathrm{m}}(\vartheta, \vartheta, \mathrm{t})$ and $\mathrm{F}_{\mathrm{m}}(\vartheta, \vartheta, \mathrm{t})=\mathrm{F}_{\mathrm{m}}(\mathrm{T} \vartheta, \mathrm{T} \vartheta, \mathrm{t})$

then $\mathrm{T} \vartheta=\vartheta$. Furthermore, if $\vartheta$ and $\vartheta^{*}$ are two fixed points then $\left(\vartheta, \vartheta^{*}\right) \in E(\tilde{G})$. Then $\mathrm{T}$ has a unique fixed point.

Proof: Let $\vartheta_{0} \in \mathrm{X}$ be any arbitrary point. Define $\vartheta_{\mathrm{n}+1}=$ $\mathrm{T} \vartheta_{\mathrm{n}}$ and $\zeta_{\mathrm{n}}(\mathrm{t})=\mathrm{F}_{\mathrm{m}}\left(\vartheta_{\mathrm{n}}, \vartheta_{\mathrm{n}+1}, \mathrm{t}\right)$ for all $\mathrm{n} \in \mathrm{Z}^{+} \cup\{0\}$. If there exist $\mathrm{n}_{0} \in \mathrm{Z}^{+} \cup\{0\}$ such that $\vartheta_{n_{0}+1}=\vartheta_{n_{0}}$ i.e $\mathrm{T} \vartheta_{n_{0}}=\vartheta_{n_{0}}$, then $\vartheta_{n_{0}}$ is a fixed point. Thus for each n, suppose that $0<$ $\zeta_{\mathrm{n}}(\mathrm{t})<1$ that is, $\vartheta_{\mathrm{n}} \neq \vartheta_{\mathrm{n}+1}$ for each $n$. From (14), we can write

$$
\begin{gathered}
\psi\left(\zeta_{\mathrm{n}}(\mathrm{t})\right)=\psi\left(\mathrm{F}_{\mathrm{m}}\left(\vartheta_{\mathrm{n}}, \vartheta_{\mathrm{n}+1}, \mathrm{t}\right)\right)=\psi\left(\mathrm{F}_{\mathrm{m}}\left(\mathrm{T} \vartheta_{\mathrm{n}-1}, \mathrm{~T} \vartheta_{\mathrm{n}}, \mathrm{t}\right)\right) \leq \\
\mathrm{K}(\mathrm{t}) . \psi\left(\zeta_{\mathrm{n}-1}(\mathrm{t})\right)<\psi\left(\zeta_{\mathrm{n}-1}(\mathrm{t})\right) .
\end{gathered}
$$

It follows that for every $\mathrm{t}>0,\left\{\zeta_{\mathrm{n}}(\mathrm{t})\right\}$ is increasing sequence with respect to $\mathrm{n}$ since $\psi$ is strictly decreasing function and also it is bounded, so it converges to $\zeta(t)$ (let say) and $\zeta_{\mathrm{n}}(\mathrm{t}) \leq \zeta(\mathrm{t})$.

Suppose that $0<\zeta(\mathrm{t})<1$. By (15), we have

$$
\psi\left(\zeta_{\mathrm{n}+1}(\mathrm{t})\right) \leq \kappa(\mathrm{t}) \cdot \psi\left(\zeta_{\mathrm{n}}(\mathrm{t})\right) .
$$

Letting as $\mathrm{n} \rightarrow \infty$ for each $\mathrm{t}>0$, since $\psi$ is left continuous, we get

$$
\begin{aligned}
& \psi(\zeta(\mathrm{t})) \leq \mathrm{K}(\mathrm{t}) \cdot \psi(\zeta(\mathrm{t})) \\
& \Rightarrow(1-\mathrm{K}(\mathrm{t})) \psi(\zeta(\mathrm{t})) \leq 0
\end{aligned}
$$

implies that $\psi(\zeta(\mathrm{t}))=0$. Hence $\zeta(\mathrm{t})=1$. Therefore, $\lim _{n \rightarrow \infty} \zeta_{n}(\mathrm{t})$ $=1$.

Now, we claim that $\left\{\boldsymbol{\vartheta}_{\mathrm{n}}\right\}$ is a Cauchy sequence.

By assumption (b), $\lim _{n, m \rightarrow \infty}\left[M_{\vartheta_{n}, \vartheta_{m}, t}-m_{\vartheta_{n}, \vartheta_{m}, t}\right]=0$. Suppose that if it is not a Cauchy sequence then there exist $\mathrm{k}_{0} \in \mathrm{Z}^{+} \mathrm{U}\{0\}$ such that $\mathrm{F}_{\mathrm{m}}\left(\vartheta_{\mathrm{n}}, \vartheta_{\mathrm{m}}, \mathrm{t}\right) \leq M_{\vartheta_{n}, \vartheta_{m}, t}-\varepsilon$ for all $\mathrm{m}, \mathrm{n} \geq \mathrm{k}_{0}$. For any $l \in \mathrm{N}$, we obtain that

$$
\begin{gathered}
\mathrm{F}_{\mathrm{m}}\left(\vartheta_{k_{0}+l+2}, \vartheta_{k_{0}+l+1}, \mathrm{t}\right) \leq \mathrm{M}_{\vartheta_{k_{0}+l+2,} \vartheta_{k_{0}+l+1, t}}-\varepsilon \leq \\
1-\varepsilon .
\end{gathered}
$$

By applying (14) and using definition of $\psi$ (as it is monotonic), it can be easily prove that the sequence $\left\{\mathrm{F}_{\mathrm{m}}\left(\vartheta_{k_{0}+l+2}, \vartheta_{k_{0}+l+1}, \mathrm{t}\right)\right\}$ is monotonic and bounded sequence with respect to $\mathrm{p}$.

We put $\lim _{n \rightarrow \infty} \mathrm{F}_{\mathrm{m}}\left(\vartheta_{k_{0}+l+2}, \vartheta_{k_{0}+l+1}, \mathrm{t}\right)=\rho$ for all $\mathrm{t}>0$ and $\rho \in(0,1-\varepsilon]$

since $\mathrm{F}_{\mathrm{m}}\left(\vartheta_{k_{0}+l+2}, \vartheta_{k_{0}+l+1}, \mathrm{t}\right) \leq 1-\varepsilon$. Hence, we have

$\psi\left(\mathrm{F}_{\mathrm{m}}\left(\vartheta_{k_{0}+l+2}, \vartheta_{k_{0}+l+1}, \mathrm{t}\right)\right) \leq \mathrm{K}(\mathrm{t}) . \psi\left(\mathrm{F}_{\mathrm{m}}\left(\vartheta_{k_{0}+l+1}\right.\right.$, $\left.\left.\vartheta_{k_{0}+l}, \mathrm{t}\right)\right)$.

Taking $l \rightarrow \infty$, we obtain 
$\psi(\rho) \leq \mathrm{K}(\mathrm{t}) . \psi(\rho)<\psi(\rho)$ and $\psi(\rho)=0$ iff $\rho=1$ implies that $\psi(\rho) \leq 0$ which leads to a contradiction.

Now, we take another case, there exist $0<\varepsilon<1$, and two sequences $\{x(n)\}$ and $\{y(n)\}$ such that for every $n \in Z^{+}$ $U\{0\}$ and $t>0$, we get

$$
\begin{aligned}
& \mathrm{x}(\mathrm{n})>\mathrm{y}(\mathrm{n})>\mathrm{n}, \mathrm{F}_{\mathrm{m}}\left(\vartheta_{x(\mathrm{n})}, \vartheta_{\mathrm{y}(\mathrm{n})}, \mathrm{t}\right) \leq \mathrm{M}_{\vartheta_{\mathrm{x}(\mathrm{n}),} \vartheta_{\mathrm{y}(\mathrm{n}), \mathrm{t}}}-\varepsilon \leq \\
& 1-\varepsilon \text { and } \\
& \mathrm{F}_{\mathrm{m}}\left(\vartheta_{\mathrm{x}(\mathrm{n})-1}, \vartheta_{\mathrm{y}(\mathrm{n})-1}, \mathrm{t}\right)>\mathrm{M}_{\vartheta_{\mathrm{x}(\mathrm{n})-1,} \vartheta_{\mathrm{y}(\mathrm{n})-1, t}}-\varepsilon \text { and } \mathrm{F}_{\mathrm{m}}\left(\vartheta_{\mathrm{x}(\mathrm{n})-1}\right. \text {, } \\
& \left.\vartheta_{\mathrm{y}(\mathrm{n})}, \mathrm{t}\right)>\mathrm{M}_{\vartheta_{\mathrm{x}(\mathrm{n})-1,}, \vartheta_{\mathrm{y}(\mathrm{n}), t}-\varepsilon .}
\end{aligned}
$$

For each $n \in Z^{+} \cup\{0\}$, assume that $r_{n}(t)=F_{m}\left(\vartheta_{x(n)}, \vartheta_{y(n)}\right.$, t) then by (FMM 4), we have

$$
\begin{aligned}
& \mathrm{F}_{\mathrm{m}}\left(\vartheta_{\mathrm{x}(\mathrm{n})}, \vartheta_{\mathrm{y}(\mathrm{n})}, \mathrm{t}\right) * \mathrm{M}_{\vartheta_{\mathrm{x}(\mathrm{n})}, \vartheta_{\mathrm{x}(\mathrm{n})-1,}, t} * \mathrm{M}_{\vartheta_{\mathrm{x}(\mathrm{n})-1}, \vartheta_{\mathrm{y}(\mathrm{n}), t}} \\
& \geq \mathrm{F}_{\mathrm{m}}\left(\vartheta_{\mathrm{x}(\mathrm{n})}, \vartheta_{\mathrm{x}(\mathrm{n})-1}, \mathrm{t} / 2\right) * \mathrm{~F}_{\mathrm{m}}\left(\vartheta_{\mathrm{x}(\mathrm{n})-1}, \vartheta_{\mathrm{y}(\mathrm{n})}, \mathrm{t} / 2\right) * \\
& \mathrm{M}_{\vartheta_{\mathrm{x}(\mathrm{n})}, \vartheta_{\mathrm{y}(\mathrm{n}), t}}
\end{aligned}
$$

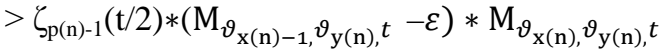

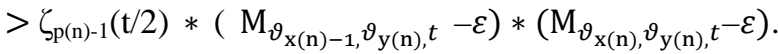$$
\text { As } \quad \mathrm{M}_{\vartheta_{\mathrm{x}(\mathrm{n}),} \vartheta_{\mathrm{x}(\mathrm{n})-1, \mathrm{t}}} * \mathrm{M}_{\vartheta_{\mathrm{x}(\mathrm{n})-1,} \vartheta_{\mathrm{y}(\mathrm{n}), \mathrm{t}}} \geq \zeta_{\mathrm{p}(\mathrm{n})-1}(\mathrm{t} / 2) \quad *
$$

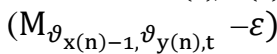$$
\text { implies that } \mathrm{F}_{\mathrm{m}}\left(\vartheta_{\mathrm{x}(\mathrm{n})}, \vartheta_{\mathrm{y}(\mathrm{n})}, \mathrm{t}\right)>\left(\mathrm{M}_{\vartheta_{\mathrm{x}(\mathrm{n})}, \vartheta_{\mathrm{y}(\mathrm{n}), \mathrm{t}}}-\varepsilon\right) \text {. }
$$

Now by (14), we obtain

$$
\begin{gathered}
\psi\left(\mathrm { F } _ { \mathrm { m } } \left(\vartheta_{\mathrm{x}(\mathrm{n})},\right.\right. \\
\left.\left.\vartheta_{\mathrm{y}(\mathrm{n})}, \mathrm{t}\right)\right) \leq \mathrm{K}(\mathrm{t}) . \psi\left(\mathrm{F}_{\mathrm{m}}\left(\vartheta_{\mathrm{x}(\mathrm{n})-1}, \vartheta_{\mathrm{y}(\mathrm{n})-1}, \mathrm{t}\right)\right) \\
<\psi\left(\mathrm{F}_{\mathrm{m}}\left(\vartheta_{\mathrm{x}(\mathrm{n})-1}, \vartheta_{\mathrm{y}(\mathrm{n})-1}, \mathrm{t}\right)\right) .
\end{gathered}
$$

By the monotonicity of $\psi$, we know that $\mathrm{F}_{\mathrm{m}}\left(\vartheta_{\mathrm{x}(\mathrm{n})}, \vartheta_{\mathrm{y}(\mathrm{n})}\right.$, t)) $>\mathrm{F}_{\mathrm{m}}\left(\vartheta_{\mathrm{x}(\mathrm{n})-1}, \vartheta_{\mathrm{y}(\mathrm{n})-1}, \mathrm{t}\right) \forall \mathrm{n}$.

Next, by using (16), we have

$$
\begin{aligned}
\mathrm{M}_{\vartheta_{\mathrm{x}(\mathrm{n})}, \vartheta_{\mathrm{y}(\mathrm{n}), t}-\varepsilon} \geq \mathrm{F}_{\mathrm{m}}\left(\vartheta_{\mathrm{x}(\mathrm{n})}, \vartheta_{\mathrm{y}(\mathrm{n})}, \mathrm{t}\right)>\mathrm{F}_{\mathrm{m}}\left(\vartheta_{\mathrm{x}(\mathrm{n})-1}, \vartheta_{\mathrm{y}(\mathrm{n})-1},\right. \\
\mathrm{t})>\mathrm{M}_{\vartheta_{\mathrm{x}(\mathrm{n})-1}, \vartheta_{\mathrm{y}(\mathrm{n})-1, t}-\varepsilon}
\end{aligned}
$$

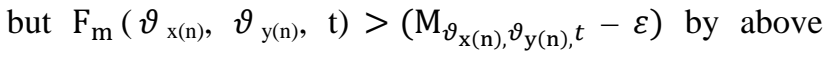
computations which leads to a contradiction.

This shows that $\left\{\vartheta_{\mathbf{n}}\right\}$ is a Cauchy sequence in the complete non-Archimedean fuzzy m-metric space X. Thus by completeness of $\mathrm{X}$, there exists a point $\vartheta \in \mathrm{X}$ such that sequence $\left\{\vartheta_{\mathrm{n}}\right\}$ converges to point $\vartheta$.

Next, we prove that $\vartheta$ is a fixed point of $\mathrm{T}$. By assumption (a), there exists a subsequence $\left\{\vartheta_{\mathrm{t}(\mathrm{n})}\right\}$ of $\left\{\vartheta_{\mathrm{n}}\right\}$ such that $\vartheta_{\mathrm{t}(\mathrm{n})} \neq \vartheta$ for each natural number $\mathrm{n}$ and $\left(\vartheta_{\mathrm{t}(\mathrm{n})}, \vartheta\right) \in E(\tilde{G})$ for all $\mathrm{n}$. From (14), we have

$$
\begin{gathered}
0 \leq \psi\left(\mathrm{F}_{\mathrm{m}}\left(\vartheta_{\mathrm{t}(\mathrm{n})+1}, \mathrm{~T} \vartheta, \mathrm{t}\right)\right)=\psi\left(\mathrm{F}_{\mathrm{m}}\left(\mathrm{T} \vartheta_{\mathrm{t}(\mathrm{n})}, \mathrm{T} \vartheta, \mathrm{t}\right)\right) \leq \\
\mathrm{K}(\mathrm{t}) \cdot \psi\left(\mathrm{F}_{\mathrm{m}}\left(\vartheta_{\mathrm{t}(\mathrm{n})}, \vartheta, \mathrm{t}\right) .\right.
\end{gathered}
$$

Letting $n \rightarrow \infty$ in (17) and by using assumption (b), Lemma 2.4, we get

$$
\begin{gathered}
0 \leq \psi\left(\mathrm{F}_{\mathrm{m}}(\vartheta, \mathrm{T} \vartheta, \mathrm{t})\right) \leq \mathrm{K}(\mathrm{t}) \psi\left(\mathrm{F}_{\mathrm{m}}(\vartheta, \vartheta, \mathrm{t}) \leq\right. \\
\mathrm{K}(\mathrm{t}) \cdot \psi\left(\mathrm{F}_{\mathrm{m}}(\vartheta, \mathrm{T} \vartheta, \mathrm{t})\right)
\end{gathered}
$$

implies that $\psi\left(\mathrm{F}_{\mathrm{m}}(\vartheta, \mathrm{T} \vartheta, \mathrm{t})\right)=0$ and by property (p2), it follows that

$\mathrm{F}_{\mathrm{m}}(\vartheta, \mathrm{T} \vartheta, \mathrm{t})=1=M_{\vartheta, T \vartheta, t}$ implies $\mathrm{T} \vartheta=\vartheta$ by assumption (b).
Next, we show that $\vartheta$ is the unique fixed point of T. For this, we consider $\vartheta^{*}(\neq \vartheta)$ is another fixed point of $\mathrm{T}$ then by (14), we get

$$
\begin{gathered}
\psi\left(\mathrm{F}_{\mathrm{m}}\left(\vartheta, \vartheta^{*}, \mathrm{t}\right)\right)=\psi\left(\mathrm{F}_{\mathrm{m}}\left(\mathrm{T} \vartheta, \mathrm{T} \vartheta^{*}, \mathrm{t}\right)\right) \leq \mathrm{K}(\mathrm{t}) \psi\left(\mathrm{F}_{\mathrm{m}}(\vartheta,\right. \\
\left.\vartheta^{*}, \mathrm{t}\right)<\psi\left(\mathrm{F}_{\mathrm{m}}\left(\vartheta, \vartheta^{*}, \mathrm{t}\right)\right.
\end{gathered}
$$

which contradict the assumption of $\psi$. Hence, $\vartheta$ is the unique fixed point of $\mathrm{T}$.

Theorem 4.2. Let $\left(\mathrm{X}, \mathrm{F}_{\mathrm{m}}, *\right)$ be a compact non-Archimedean fuzzy m-metric space with graph $G=(V$, E) and $T$ be a G-continuous self-map of $X$ satisfies the following condition:

$$
\psi\left(\mathrm{F}_{\mathrm{m}}(\mathrm{T} \vartheta, \mathrm{T} \rho, \mathrm{t})\right)<\psi\left(\mathrm{F}_{\mathrm{m}}(\vartheta, \rho, \mathrm{t})\right)
$$

where $\psi \in \Psi$ and $\vartheta, \rho \in E(\tilde{G}), \vartheta \neq \rho$, for any $\mathrm{t}>0$. In addition, assume that there exist a subsequence $\left\{\vartheta_{\mathrm{q}(\mathrm{n})}\right\}$ of $\left\{\vartheta_{\mathrm{n}}\right\}$ such that $\lim _{n \rightarrow \infty} \mathrm{M}_{\vartheta_{\mathrm{q}(\mathrm{n}), \vartheta} \mathrm{q}(\mathrm{n})+1, \mathrm{t}}=M_{\vartheta, T \vartheta, t}=\mathrm{M}_{\mathrm{T} \vartheta, \mathrm{T}^{2} \vartheta, \mathrm{t}}$ for all $\mathrm{n}$ with whenever a subsequence $\left\{\vartheta_{\mathrm{q}(\mathrm{n})}\right\}$ of $\left\{\vartheta_{\mathrm{n}}\right\}$ converges to $\vartheta$ with $\left(\vartheta_{\mathrm{q}(\mathrm{n})}, \vartheta_{\mathrm{q}(\mathrm{n})+1}\right) \in E(\tilde{G})$. Furthermore, if $\vartheta$ and $\vartheta^{*}$ are two fixed points then $(\vartheta, \vartheta *) \in E(\tilde{G})$. Then $\mathrm{T}$ has a unique fixed point.

Proof. Let $\vartheta_{0} \in \mathrm{X}$ be any arbitrary number. Define a sequence $\left\{\vartheta_{\mathrm{n}}\right\}$ such that $\vartheta_{\mathrm{n}}=\mathrm{T} \vartheta_{\mathrm{n}-1}=\mathrm{T}^{\mathrm{n}} \vartheta_{\mathrm{o}}$, with $\mathrm{n} \in \mathrm{N} \mathrm{U}$ $\{0\}$. If $\mathrm{T}^{\mathrm{n}} \vartheta_{\mathrm{o}}=\mathrm{T}^{\mathrm{n}+1} \vartheta_{\mathrm{o}}$ for some $\mathrm{n}$, then there exists $\mathrm{p} \in \mathrm{X}$ such that $\mathrm{p}=\mathrm{T}^{\mathrm{q}} \vartheta_{\mathrm{o}}$ for all $\mathrm{q} \geq \mathrm{n}$. So we assume that $\mathrm{T}^{\mathrm{n}} \vartheta_{\mathrm{o}}$ $\neq \mathrm{T}^{\mathrm{n}+1} \vartheta_{\mathrm{o}}$ for every $\mathrm{n}$. As $\left(\mathrm{X}, \mathrm{F}_{\mathrm{m}}, *\right)$ is a compact fuzzy m-metric space, so there exist a subsequence $\left\{\mathrm{T}^{\mathrm{q}(\mathrm{n})} \vartheta_{0}\right\}$ of $\left\{\mathrm{T}^{\mathrm{n}} \vartheta_{\mathrm{o}}\right\}$ such that $\vartheta_{\mathrm{q}(\mathrm{n})}=\mathrm{T}^{\mathrm{q}(\mathrm{n})} \vartheta_{\mathrm{o}} \rightarrow \vartheta \in \mathrm{X}$ as $\mathrm{n} \rightarrow \infty$.

By the continuity of $\mathrm{T}$, it follows that

$$
\lim _{n \rightarrow \infty} \vartheta_{q(\mathrm{n})+1}=\mathrm{T}(\vartheta) \text { and } \lim _{n \rightarrow \infty} \vartheta_{q(\mathrm{n})+2}=\mathrm{T}^{2}(\vartheta) \text {. }
$$

Since $\psi$ is left continuous and by using assumption and Lemma 2.3, we get

$\psi\left(\mathrm{F}_{\mathrm{m}}(\vartheta, \mathrm{T} \vartheta, \mathrm{t})\right)=\lim _{n \rightarrow \infty} \psi\left(\mathrm{F}_{\mathrm{m}}\left(\vartheta_{\mathrm{q}(\mathrm{n})}, \vartheta_{\mathrm{q}(\mathrm{n})+1}, \mathrm{t}\right)\right)=\lim _{n \rightarrow \infty} \psi$

$\left(\mathrm{F}_{\mathrm{m}}\left(\vartheta_{\mathrm{q}(\mathrm{n})+1}, \vartheta_{\mathrm{q}(\mathrm{n})+2}, \mathrm{t}\right)\right)=\psi\left(\mathrm{F}_{\mathrm{m}}\left(\mathrm{T} \vartheta, \mathrm{T}^{2} \vartheta, \mathrm{t}\right)\right)$.

Now we show that $\mathrm{T} \vartheta=\vartheta$. Otherwise by (18), we can obtain

$$
\psi\left(\mathrm{F}_{\mathrm{m}}\left(\mathrm{T} \vartheta, \mathrm{T}^{2} \vartheta, \mathrm{t}\right)\right)<\psi\left(\mathrm{F}_{\mathrm{m}}(\vartheta, \mathrm{T} \vartheta, \mathrm{t})\right)
$$

which is contrary to the formula (20). Thus $\mathrm{T} \vartheta=\vartheta$. The proof of uniqueness is similar to that of Theorem 4.1.

Corollary 4.1. Let $\left(\mathrm{X}, \mathrm{F}_{\mathrm{m}}, *\right)$ be a compact non-Archimedean fuzzy metric space with graph $\mathrm{G}=(\mathrm{V}, \mathrm{E})$ and $\mathrm{T}$ a continuous self-map of $\mathrm{X}$. Furthermore, let $\mathrm{K}$ be a function from $(0, \infty)$ into $(0,1)$. If for any $t>0$, T satisfies the following condition:

$$
\psi\left(\mathrm{F}_{\mathrm{m}}(\mathrm{T} \vartheta, \mathrm{T} \rho, \mathrm{t})\right) \leq \mathrm{K}(\mathrm{t}) \cdot \psi\left(\mathrm{F}_{\mathrm{m}}(\vartheta, \rho, \mathrm{t})\right)
$$

where $\psi \in \Psi$ and $\vartheta, \rho \in E(\tilde{G}), \vartheta \neq \rho$.

In addition, assume that there exists a subsequence $\left\{\vartheta_{\mathrm{q}(\mathrm{n})}\right\}$

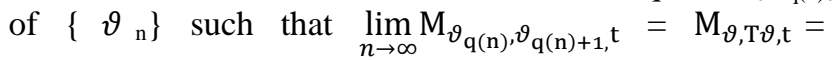
$\mathrm{M}_{\mathrm{T} \vartheta, \mathrm{T}^{2} \vartheta, \mathrm{t}}$ for all $\mathrm{n}$ with whenever a subsequence $\left\{\vartheta_{\mathrm{q}(\mathrm{n})}\right\}$ of $\left\{\vartheta_{\mathrm{n}}\right\}$ converges to $\vartheta$ with $\left(\vartheta_{\mathrm{q}(\mathrm{n}),} \vartheta_{\mathrm{q}(\mathrm{n})+1}\right) \in E(\tilde{G})$. Furthermore, if $\vartheta$ and $\vartheta^{*}$ are two fixed points then 
$\left(\vartheta, \vartheta^{*}\right) \in E(\tilde{G})$. Thus $\mathrm{T}$ has a unique fixed point.

Proof. It can be easily proved by the same method as was employed in Theorem 4.2.

Let $\left(\mathrm{X}, \mathrm{F}_{\mathrm{m}}, *\right)$ be a non-Archimedean fuzzy m- metric space and define $\mathrm{h}_{\mathrm{n}}(\mathrm{t})=\delta_{F_{m}}\left(\mathrm{~A}_{\mathrm{n}}, \mathrm{t}\right)$ where

$\delta_{F_{m}}\left(\mathrm{~A}_{\mathrm{n}}, \mathrm{t}\right)=\inf \left\{\mathrm{F}_{\mathrm{m}}(\vartheta, \rho, \mathrm{t}): \vartheta, \rho \in \mathrm{A}_{\mathrm{n}}\right\}$ and $A_{n}=\left\{\vartheta_{n}, \vartheta_{n+1}, \vartheta_{n+2}, \ldots \ldots ..\right\}$ in fuzzy m- metric space then clearly, we can observe that $h_{n}(t)$ is finite for all natural numbers $n$ and $0 \leq \mathrm{h}_{\mathrm{n}}(\mathrm{t}) \leq 1, \mathrm{~h}_{\mathrm{n}}(\mathrm{t})$ is non-increasing , $\mathrm{h}_{\mathrm{n}}(\mathrm{t}) \rightarrow \mathrm{h}(\mathrm{t})$ for some $0 \leq \mathrm{h}(\mathrm{t}) \leq 1$ and also $\mathrm{h}_{\mathrm{n}}(\mathrm{t}) \leq \mathrm{F}_{\mathrm{m}}\left(\vartheta_{l}, \vartheta_{\mathrm{m}}, \mathrm{t}\right)$ for all $l, \mathrm{~m} \geq \mathrm{n}$.

Define a collection $\Phi$ of functions $\mathrm{F}$ from $[0,1]^{3} \times[0,1]$ into $[-1,1]$ satisfying the following conditions:

1. $\mathrm{F}$ is continuous on $[0,1]^{3} \times[0,1]$;

2. $\quad \mathrm{F}$ is non-decreasing on $[0,1]^{3}$;

3. $\mathrm{F}((\zeta, \zeta, \zeta), \kappa) \leq 0$ implies that $\kappa \geq \gamma(\zeta)$ where $\gamma$ : $[0,1] \rightarrow[0,1]$ is a nondecreasing continuous function with $\gamma(\varsigma)>\varsigma$ for $\varsigma \in[0,1)$.

Theorem 4.3. Let $\left(\mathrm{X}, \mathrm{F}_{\mathrm{m}}, *\right)$ be a complete non-Archimedean fuzzy $\mathrm{m}$ - metric space and $\mathrm{T}$ be a self-map of X satisfying

$$
\mathrm{F}\left(\mathrm{F}_{\mathrm{m}}(\vartheta, \rho, \mathrm{t}), \mathrm{F}_{\mathrm{m}}(\mathrm{T} \vartheta, \vartheta, \mathrm{t}), \mathrm{F}_{\mathrm{m}}(\mathrm{T} \vartheta, \rho, \mathrm{t}), \mathrm{F}_{\mathrm{m}}(\mathrm{T} \vartheta, \mathrm{T} \rho, \mathrm{t})\right)
$$

for all $\vartheta, \rho \in E(\tilde{G})$, where $\mathrm{F} \in \Phi$. In addition assume that if a sequence $\vartheta_{\mathrm{n}}=\mathrm{T} \vartheta_{\mathrm{n}-1}$ then $\left(\vartheta_{\mathrm{n}}, \vartheta_{\mathrm{m}}\right) \in E(\tilde{G})$ for all $\mathrm{n}, \mathrm{m}$ and suppose that sequence $\left\{\mathrm{F}_{\mathrm{m}}\left(\vartheta_{\mathrm{n}}, \vartheta_{\mathrm{n}}, \mathrm{t}\right)\right\}$ is constant. Also, if $\vartheta_{\mathrm{n}} \rightarrow \vartheta$ then $\left(\vartheta_{\mathrm{n}}, \vartheta\right) \in E(\widetilde{G})$ and $\mathrm{F}_{\mathrm{m}}(\vartheta, \vartheta, \mathrm{t})$ $=\mathrm{F}_{\mathrm{m}}(\mathrm{T} \vartheta, \mathrm{T} \vartheta, \mathrm{t})$. Further, if $\vartheta_{1}$ and $\vartheta_{2}$ be two fixed points with $\mathrm{F}_{\mathrm{m}}\left(\vartheta_{1}, \vartheta_{1}, \mathrm{t}\right)=\mathrm{F}_{\mathrm{m}}\left(\vartheta_{2}, \vartheta_{2}, \mathrm{t}\right)$ then $\left(\vartheta_{1}, \vartheta_{2}\right) \in E(\tilde{G})$. Then $\mathrm{T}$ has a unique fixed point in $\mathrm{X}$.

Proof: Let $\vartheta_{0} \in \mathrm{X}$ be an arbitrary element and take a sequence $\vartheta_{\mathrm{n}+1}=\mathrm{T} \vartheta_{\mathrm{n}}$. Let $\mathrm{h}_{\mathrm{n}}(\mathrm{t})=\delta_{M}\left(\mathrm{~A}_{\mathrm{n}}, \mathrm{t}\right)$ where $\mathrm{A}_{\mathrm{n}}=\left\{\vartheta_{\mathrm{n}}, \vartheta_{\mathrm{n}+1}, \ldots ..\right\}$. Since $\mathrm{h}_{\mathrm{n}}(\mathrm{t}) \rightarrow \mathrm{h}(\mathrm{t})$ as $n \rightarrow \infty$ for some $0 \leq h(t) \leq 1$. If for some $n \in Z^{+}, \vartheta_{n}=\vartheta_{n+1}$ then $T$ has a fixed point. Therefore, suppose that $\vartheta_{\mathrm{n}} \neq \vartheta_{\mathrm{n}+1}$ for each $\mathrm{n}$ $\in \mathrm{Z}^{+}$. Let $\mathrm{k}$ be any fixed natural number. For $\vartheta=\vartheta_{\mathrm{n}-1}$, $\rho=\vartheta_{\mathrm{n}+\mathrm{m}-1,}$, (22) becomes where $\mathrm{n} \geq \mathrm{k}$ and $\mathrm{m} \in \mathrm{N}$, we have

$\mathrm{F}\left(\mathrm{F}_{\mathrm{m}}\left(\vartheta_{\mathrm{n}-1}, \vartheta_{\mathrm{n}+\mathrm{m}-1}, \mathrm{t}\right), \mathrm{F}_{\mathrm{m}}\left(\mathrm{T} \vartheta_{\mathrm{n}-1}, \vartheta_{\mathrm{n}-1}, \mathrm{t}\right), \mathrm{F}_{\mathrm{m}}\left(\mathrm{T} \vartheta_{\mathrm{n}-1}, \vartheta_{\mathrm{n}+\mathrm{m}-1}, \mathrm{t}\right)\right.$, $\left.\mathrm{F}_{\mathrm{m}}\left(\mathrm{T} \vartheta_{\mathrm{n}-1}, \mathrm{~T} \vartheta_{\mathrm{n}+\mathrm{m}-1}, \mathrm{t}\right)\right)$

$=F\left(F_{m}\left(\vartheta_{n-1}, \vartheta_{n+m-1}, t\right), F_{m}\left(\vartheta_{n}, \vartheta_{n-1}, t\right), F_{m}\left(\vartheta_{n}, \vartheta_{n+m-1}, t\right)\right.$, $\left.F_{m}\left(\vartheta_{n}, \vartheta_{n+m}, t\right)\right) \leq 0$.

Next using definition of $\mathrm{h}_{\mathrm{n}}(\mathrm{t})$, we get

$F\left(h_{n-1}(t), h_{n-1}(t), h_{n}(t), F_{m}\left(\vartheta_{n}, \vartheta_{n+m}, t\right)\right) \leq 0$.

As $h_{n}(t)$ is non- increasing and $F$ is non-decreasing on [0, $1]^{3}$, therefore the above inequality becomes

$F\left(h_{k-1}(t), h_{k-1}(t), h_{k-1}(t), F_{m}\left(\vartheta_{n}, \vartheta_{n+m}, t\right)\right) \leq 0$.

That is,

$\mathrm{F}_{\mathrm{m}}\left(\vartheta_{\mathrm{n}}, \vartheta_{\mathrm{n}+\mathrm{m}}, \mathrm{t}\right) \geq \gamma\left(\mathrm{f}_{\mathrm{k}-1}(\mathrm{t})\right)$.

Thus, for all $n \geq \mathrm{k}$, it follows that $\left.\min _{n \geq \mathrm{k},}\left\{\mathrm{F}_{\mathrm{m}}\left(\vartheta_{n}, \vartheta_{n+m}, t\right)\right\}=\mathrm{h}_{\mathrm{k}}(\mathrm{t})\right) \geq \gamma\left(\mathrm{h}_{\mathrm{k}-1}(\mathrm{t})\right)$.

Taking as $\mathrm{k} \rightarrow \infty$, so we have $\mathrm{h}(\mathrm{t}) \geq \gamma(\mathrm{h}(\mathrm{t}))$. If $\mathrm{h}(\mathrm{t}) \neq 1$ then $f(t) \geq \gamma(f(t))>h(t)$, which is a contradiction. Thus $\mathrm{f}(\mathrm{t})=1$ and hence $\lim _{n \rightarrow \infty} \mathrm{h}_{\mathrm{n}}(\mathrm{t})=1$. Thus given $\epsilon>0$, there exist an $\mathrm{N}_{0} \in \mathrm{Z}^{+}$such that $\mathrm{h}_{\mathrm{n}}(\mathrm{t})>1-\epsilon$ for all $\mathrm{n} \geq \mathrm{N}_{0}$. Then we have for $\mathrm{n} \geq \mathrm{N}_{0}$ and $\mathrm{m} \in \mathrm{N}$,

$$
\mathrm{F}_{\mathrm{m}}\left(\vartheta_{n}, \vartheta_{n+m}, t\right)>1-\epsilon>M_{\vartheta_{n,}, \vartheta_{n+m}, t}-\epsilon .
$$

By assumption and (23), $\left\{\vartheta_{n}\right\}$ is a Cauchy sequence in $X$. By the definition of $\mathrm{X}$, there exists a $\mathrm{z} \in \mathrm{X}$ such that

$$
\lim _{n \rightarrow \infty} \mathrm{F}_{\mathrm{m}}\left(\vartheta_{\mathrm{n}}, \mathrm{z}, \mathrm{t}\right)-M_{\vartheta_{n}, z, t}=0 .
$$

Again for $\vartheta=\vartheta_{\mathrm{n}}, \rho=\mathrm{z}$ in (22), then (22) becomes

$\mathrm{F}\left(\mathrm{F}_{\mathrm{m}}\left(\vartheta_{\mathrm{n}}, \mathrm{z}, \mathrm{t}\right), \mathrm{F}_{\mathrm{m}}\left(\mathrm{T} \vartheta_{\mathrm{n}}, \vartheta_{\mathrm{n}}, \mathrm{t}\right), \mathrm{F}_{\mathrm{m}}\left(\mathrm{T} \vartheta_{\mathrm{n}}, \mathrm{z}, \mathrm{t}\right), \mathrm{F}_{\mathrm{m}}\left(\mathrm{T} \vartheta_{\mathrm{n}}\right.\right.$, $\mathrm{Tz}, \mathrm{t}))$

$=F\left(F_{m}\left(\vartheta_{n}, z, t\right), F_{m}\left(\vartheta_{n+1}, \vartheta_{n}, t\right), F_{m}\left(\vartheta_{n+1}, z, t\right), F_{m}\left(\vartheta_{n+1}\right.\right.$, $\mathrm{Tz}, \mathrm{t})) \leq 0$.

Hence using (23), we have

$\lim _{n \rightarrow \infty} \sup F\left(F_{m}\left(\vartheta_{n}, z, t\right), F_{m}\left(\vartheta_{n+1}, \vartheta_{n}, t\right), F_{m}\left(\vartheta_{n+1}, z, t\right)\right.$, $\left.F_{m}\left(\vartheta_{n+1}, T z, t\right)\right)$

$=\mathrm{F}\left(\lim _{n \rightarrow \infty} \sup M_{\vartheta_{n}, z, t}, 1, \quad \lim _{n \rightarrow \infty} \sup M_{\vartheta_{n}, z, t}, \lim _{n \rightarrow \infty} \sup \right.$ $\left.\mathrm{F}_{\mathrm{m}}\left(\vartheta_{\mathrm{n}+1}, \mathrm{Tz}, \mathrm{t}\right)\right) \leq 0$.

or F(lim $\lim _{n \rightarrow \infty} \sup M_{\vartheta_{n}, z, t}, \lim _{n \rightarrow \infty} \sup M_{\vartheta_{n}, z, t}, \lim _{n \rightarrow \infty} \sup M_{\vartheta_{n}, z, t}$, $\left.\lim _{n \rightarrow \infty} \sup F_{m}\left(\vartheta_{n+1}, T z, t\right)\right)$

$\leq \mathrm{F}\left(\lim _{n \rightarrow \infty} \sup M_{\vartheta_{n}, z, t}, 1, \lim _{n \rightarrow \infty} \sup M_{\vartheta_{n}, z, t}, \lim _{n \rightarrow \infty} \sup \mathrm{F}_{\mathrm{m}}\right.$ $\left.\left(\vartheta_{\mathrm{n}+1}, \mathrm{Tz}, \mathrm{t}\right)\right) \leq 0$ implies that

$\lim _{n \rightarrow \infty} \sup M_{\vartheta_{n}, T z, t}=\lim _{n \rightarrow \infty} \sup M_{\vartheta_{n+1}, T z, t} \geq \lim _{n \rightarrow \infty} \sup \mathrm{F}_{\mathrm{m}}(\vartheta$

$$
\mathrm{n}+1, \mathrm{Tz}, \mathrm{t}) \geq \gamma\left(\lim _{n \rightarrow \infty} \sup M_{\vartheta_{n}, z, t}\right)
$$

$\Rightarrow \lim _{n \rightarrow \infty} \sup M_{\vartheta_{n}, T z, t} \geq \gamma\left(\lim _{n \rightarrow \infty} \sup \quad M_{\vartheta_{n}, T z, t}\right)$ (by assumption).

This implies that $\lim _{n \rightarrow \infty} \sup M_{\vartheta_{n}, T z, t}=1$.

Again by (24), we have

$\mathrm{F}_{\mathrm{m}}(\mathrm{z}, \mathrm{Tz}, \mathrm{t}) \geq \lim _{n \rightarrow \infty} \sup \mathrm{F}_{\mathrm{m}}\left(\vartheta_{\mathrm{n}+1}, \mathrm{Tz}, \mathrm{t}\right) \geq \gamma\left(\lim _{n \rightarrow \infty} \sup \right.$ $\left.M_{\vartheta_{n}, z, t}\right)=\gamma(1)=1$.

That is, we have $\mathrm{F}_{\mathrm{m}}(\mathrm{z}, \mathrm{Tz}, \mathrm{t})=1=\mathrm{M}_{\mathrm{z}, \mathrm{Tz}, \mathrm{t}}$ implies that $\mathrm{Tz}$ $=\mathrm{z}$ and $\mathrm{F}_{\mathrm{m}}(\mathrm{z}, \mathrm{z}, \mathrm{t})=1$.

To prove uniqueness part, we consider $\vartheta_{1}$ and $\vartheta_{2}$ be two fixed points of T. For $\vartheta=\vartheta_{1}, \rho=\vartheta_{2}$ (22) becomes,

$\mathrm{F}\left(\mathrm{F}_{\mathrm{m}}\left(\vartheta_{1}, \vartheta_{2}, \mathrm{t}\right), \mathrm{F}_{\mathrm{m}}\left(\mathrm{T} \vartheta_{1}, \vartheta_{1}, \mathrm{t}\right), \mathrm{F}_{\mathrm{m}}\left(\mathrm{T} \vartheta_{1}, \vartheta_{2}, \mathrm{t}\right), \mathrm{F}_{\mathrm{m}}\left(\mathrm{T} \vartheta_{1}\right.\right.$, $\left.\mathrm{T} \vartheta_{2}, \mathrm{t}\right)$ )

$=F\left(F_{m}\left(\vartheta_{1}, \vartheta_{2}, t\right), F_{m}\left(\vartheta_{1}, \vartheta_{1}, t\right), F_{m}\left(\vartheta_{1}, \vartheta_{2}, t\right), F_{m}\left(\vartheta_{1}\right.\right.$, $\left.\left.\vartheta_{2}, \mathrm{t}\right)\right) \leq 0$.

Since $\mathrm{F}$ is non-decreasing on $[0,1]^{3}$, then it follows that $\mathrm{F}\left(\mathrm{F}_{\mathrm{m}}\left(\vartheta_{1}, \vartheta_{2}, \mathrm{t}\right), \mathrm{F}_{\mathrm{m}}\left(\vartheta_{1}, \vartheta_{2}, \mathrm{t}\right), \mathrm{F}_{\mathrm{m}}\left(\vartheta_{1}, \vartheta_{2}, \mathrm{t}\right), \mathrm{F}_{\mathrm{m}}\left(\vartheta_{1}\right.\right.$, $\left.\left.\vartheta_{2}, \mathrm{t}\right)\right)$

$=\mathrm{F}\left(\mathrm{F}_{\mathrm{m}}\left(\vartheta_{1}, \vartheta_{2}, \mathrm{t}\right), 1, \mathrm{~F}_{\mathrm{m}}\left(\vartheta_{1}, \vartheta_{2}, \mathrm{t}\right), \mathrm{F}_{\mathrm{m}}\left(\vartheta_{1}, \vartheta_{2}, \mathrm{t}\right)\right) \leq 0$

which gives that

$\mathrm{F}_{\mathrm{m}}\left(\vartheta_{1}, \vartheta_{2}, \mathrm{t}\right) \geq \gamma\left(\mathrm{F}_{\mathrm{m}}\left(\vartheta_{1}, \vartheta_{2}, \mathrm{t}\right)\right)>\mathrm{F}_{\mathrm{m}}\left(\vartheta_{1}, \vartheta_{2}, \mathrm{t}\right)$ 
which is a contradiction.

Thus, we have $\mathrm{F}_{\mathrm{m}}\left(\vartheta_{1}, \vartheta_{2}, \mathrm{t}\right)=1=M_{\vartheta_{1}, \vartheta_{2}, t}$ therefore $\vartheta_{1}=\vartheta_{2}$. Hence, $\mathrm{T}$ has a unique fixed point.

In theorem 4.3 , if we take $\mathrm{F}\left(\left(\vartheta_{1}, \vartheta_{2}, \vartheta_{3}\right), \vartheta_{4}\right)=$ $\gamma\left(\min \left\{\vartheta_{1}, \vartheta_{2}, \vartheta_{3}\right\}\right)-\vartheta_{4}$ then we obtain our next result.

Corollary 4.2. Let $\left(\mathrm{X}, \mathrm{F}_{\mathrm{m}}, *\right)$ be a complete non-Archimedean fuzzy m-metric space and $\mathrm{T}$ be a self-map of $X$ satisfying

$$
\begin{gathered}
\mathrm{F}_{\mathrm{m}}(\mathrm{T} \vartheta, \mathrm{T} \rho, \mathrm{t}) \geq \gamma\left(\operatorname { m i n } \left\{\mathrm{F}_{\mathrm{m}}(\vartheta, \rho, \mathrm{t}),\right.\right. \\
\left.\left.\mathrm{F}_{\mathrm{m}}(\mathrm{T} \vartheta, \vartheta, \mathrm{t}), \mathrm{F}_{\mathrm{m}}(\mathrm{T} \vartheta, \rho, \mathrm{t})\right\}\right)
\end{gathered}
$$

for all $\vartheta, \rho \in E(\tilde{G})$, where $\mathrm{F} \in \Phi$. In addition assume that if a sequence $\vartheta_{\mathrm{n}}=\mathrm{T} \vartheta_{\mathrm{n}-1}$ then $\mathrm{F}_{\mathrm{m}}\left(\vartheta_{\mathrm{n}}, \vartheta_{\mathrm{n}}, \mathrm{t}\right)=\mathrm{F}_{\mathrm{m}}\left(\vartheta_{\mathrm{m}}, \vartheta_{\mathrm{m}}\right.$, t) and $\left(\vartheta_{\mathrm{n}}, \vartheta_{\mathrm{m}}\right) \in E(\tilde{G})$ for all $\mathrm{n}, \mathrm{m}$. Also, if $\vartheta_{\mathrm{n}} \rightarrow \vartheta$ then $\left(\vartheta_{\mathrm{n}}, \vartheta\right) \in E(\tilde{G})$ and $\mathrm{F}_{\mathrm{m}}(\vartheta, \vartheta, \mathrm{t})=\mathrm{F}_{\mathrm{m}}(\mathrm{T} \vartheta, \mathrm{T} \vartheta, \mathrm{t})$. Further, if $\mathrm{z}$, w be two fixed points with $\mathrm{F}_{\mathrm{m}}(\mathrm{z}, \mathrm{z}, \mathrm{t})=\mathrm{F}_{\mathrm{m}}(\mathrm{w}, \mathrm{w}, \mathrm{t})$ then $(\mathrm{z}$, $\mathrm{w}) \in E(\tilde{G})$. Then $\mathrm{T}$ has a unique fixed point in $\mathrm{X}$.

\section{Conclusions}

In this paper, we proposed a generalized version of partial fuzzy metric space named as non-Archimedean fuzzy m-metric space. Further, we gave some examples and defined all its topological properties. Finally, we presented some fixed theorems in context of complete non-Archimedean fuzzy m-metric space and compact non-Archimedean fuzzy m-metric space.

\section{Funding}

This research received no external funding.

\section{Conflicts of Interest}

The authors declare no conflict of interest.

\section{REFERENCES}

[1] A. George and P. Veeramani, On some results in fuzzy metric spaces, Fuzzy Sets and Systems, vol. 64, no. 3, pp. 395-399, 1994.

[2] A. Razani, A contraction theorem in fuzzy metric spaces, Fixed Point Theory Appl., 2005.

[3] D. Michet, On the existence and the uniqueness of fixed points of Sehgal contractions, Fuzzy Sets and Systems, 156, 135-141. 2005.

[4] D. Mihet, Fuzzy- contractive mappings in non-Archimedean fuzzy metric spaces. Fuzzy Sets and Systems 159,739-744, 2008.

[5] D. Michet, A bnach contraction theorem in fuzzy metric spaces, Fuzzy Sets and System, 114, 431-439, 2004.

[6] I. Beg, C. Vetro, D. Gopal, \& M. Imdad, $(\varphi, \psi)$-weak contractions in intuitionistic fuzzy metric spaces, Journal of Intelligent and Fuzzy Systems, 26, 2497-2504, 2014.

[7] I. Kramosil and J. Michalek, Fuzzy metric and statistical metric spaces, Kybernetika, vol. 11, no. 5, pp. 336-344, 1975.

[8] I. Beg, S. Sedghi, and N. Shobe, Fixed Point Theorems in Fuzzy Metric Spaces, Int. J. Anal., vol. 2013, 2013.

[9] J. Jachymski, The contraction principle for mappings on a metric space with a graph, American mathematical society, 136 (4), 1359-1373, (2007).

[10] L. Ciric, Some new results for Banach contradiction and Edelstein contractive mappings on fuzzy metric spaces, chaos solitons fractals 42(1), pp. 146-154, 2009.

[11] L. A. Zadeh, Fuzzy sets, Information and Control, vol.8, no. 3,338-353, 1965.

[12] M. Asadi, E. Karapınar, and P. Salimi, New extension of p-metric spaces with some fixed-point results on M-metric spaces, Journal of inequality and application, 2014.

[13] M. Grabiec, Fixed points in fuzzy metric spaces, Fuzzy Sets and Systems, vol. 27, no. 3, pp. 385-389, 1988.

[14] N. Souayah, N. Mlaiki, and M. Mrad, The GM-Contraction Principle for Mappings on an M -Metric Spaces Endowed with a Graph and Fixed Point Theorems, IEEE Access, 2018.

[15] P. A. Ejegwa and A. J. Akubo O M Joshua, Intuitionistic fuzzy set and its application in career determination via normalized euclidean distance method, European Scientific Journal, 2014.

[16] R. Espinola and W. A. Krik, Fixed point theorems in Rtrees with applications to graph theory, Topology Appl., 153, 1046-1055, 2006.

[17] R. K. Saini, V. Gupta \& S. B. Singh, S. B., Fuzzy version of some fixed points theorems on expansion type maps in fuzzy metric space, Thai Journal of Mathematics, 5, 245252, 2007.

[18] S. Assaf, On M-metric spaces and fixed point theorems 2017. https://www.researchgate.net/publication/322024478.

[19] S. B. Nadler, Multivalued contraction mappings, Pacific J. Math. 30, 475-488, 1969.

[20] S. Heilpern, Fuzzy mappings and fixed point theorem, J. Math. Anal. Appl. 83, 566-569, 1981.

[21] S. Sedghi, N. Shobkolaei, and I. Altun, Partial fuzzy metric space and some fixed point results, Communications in Mathematics, 2015.

[22] S. Sharma, On fuzzy metric space, Southeast Asian Bulletin of Mathematics, 26, pp. 133-145, 2002.

[23] Y. Shen, D. Qiu, and W. Chen, Fixed point theorems in fuzzy metric spaces, Appl. Math. Lett., 2012. 